\title{
Grazing-incidence antireflection films. I. Basic theory
}

\author{
J. P. Hannon, N. V. Hung, and G. T. Trammell \\ Physics Department, Rice University, Houston, Texas 77251 \\ E. Gerdau, M. Mueller, R. Rüffer, and H. Winkler \\ Institut für Experimentalphysik, Universität Hamburg, Hamburg, Germany
}

(Received 9 May 1984)

\begin{abstract}
We discuss the possibility of a new interference technique for $\mathrm{x}$-ray and $\gamma$-ray optics-the simple idea of grazing-incidence antireflection films (GIAR films) -for creating high-efficiency antireflection coatings for near-grazing-incidence reflection of hard $\mathrm{x}$ rays and $\gamma$ rays. The motivation is the possible application to producing "ultranarrow" bandpass filters for synchrotron radiation with frequency widths $\approx 10^{-8}-10^{-6} \mathrm{eV}$, giving a unique high-resolution, long-coherence-length, $\mathrm{x}$-ray source for probing soft inelastic excitations and opening up new possibilities in x-ray interferometry. In this first of two papers on nonresonant GIAR films, we develop the basic theory and discuss in detail the simplest ideas for achieving antireflection films-impedance-matched quarter-wave films and damping stabilized solutions - which can both be obtained by coating a high-density mirror with a single lower-density film.
\end{abstract}

\section{INTRODUCTION}

Thin-film interference techniques are of central importance in modern optics. In the optical region, thin dielectric films are used to produce antireflection coatings, broad and narrow bandpass frequency filters, polarizers, dichroic mirrors for color-selective beam splitting, and multilayer high-reflectance dielectric mirrors. The technology and application of such thin-film devices have expanded dramatically during the last 30 years. ${ }^{1-6}$

In $x$-ray optics, smooth multilayer films are now being developed to produce $x$-ray mirrors for soft $x$ rays in the (100-200)-A region, with potential application to the construction of normal-incidence $\mathrm{x}$-ray reflecting telescopes and microscopes, as well as broad bandpass filters, polarizers, and beam deflectors. ${ }^{7-11}$

In neutron optics, multilayer "supermirrors" have been proposed to extend by several times the grazing-incidence critical angle $\theta_{c}$ for use in neutron polarizers and for focused mirror systems. ${ }^{12,13}$

The purpose of this paper is to discuss the possibility of a new interference technique for $\mathrm{x}$-ray and $\gamma$-ray opticsthe simple idea of grazing-incidence antireflection films (GIAR films)-for creating high-efficiency antireflection coatings for near-grazing-incidence reflection of hard $\mathrm{x}$ rays and $\gamma$ rays. ${ }^{14}$

The primary motivation for this study is the possible application to $\gamma$-ray optics: using GIAR films to eliminate the nonresonant electronic reflection from a resonant mirror offers a new technique for obtaining pure nuclear, coherent $\gamma$-ray scattering. ${ }^{14}$ If such pure resonant scattering can be achieved, an immediate application would be an "ultranarrow" bandpass filter for synchrotron radiation. The resulting beam would be highly monochromatic $\left(\Delta \omega \approx 10^{-8}-10^{-6} \mathrm{eV}\right)$ with a potential brightness greatly exceeding that available from natural $\gamma$-ray sources. Such a source would offer a unique high-resolution $x$-ray probe of "soft" inelastic excitations $\left(\Delta \omega \leqslant 10^{-6} \mathrm{eV}\right)$, opening up a new region inaccessible by other scattering methods. There would also be immediate applications to Mössbauer experiments involving coherent scattering from perfect crystals, structure determination of biomolecules, and studies of surface magnetism. Also, the long coherence length of the signal $(\approx 30 \mathrm{~m})$ could offer quite new possibilities in $\mathrm{x}$-ray interferometry.

The basic idea of GIAR films is as follows: In $x$-ray optics, the small index-of-refraction decrease $\left(N_{0}=1\right.$ $\rightarrow N_{1}=1-2 \pi \hbar^{2} n Z r_{0} \cong 1-10^{-5}$, where $n$ is the atomic density, $Z$ is the atomic number, and $\left.r_{0}=e^{2} / m c^{2}\right)$, on entering a medium of higher electron density, produces near-total reflection of $\mathrm{x}$ rays at grazing incidence on a flat surface. ${ }^{15}$ The critical angle is $\varphi_{c}=\left(n \lambda^{2} Z r_{0} / \pi\right)^{1 / 2}$. For $\varphi>\varphi_{c}$, there is a rapid dropoff in reflected intensity with increasing $\varphi$ and a rapid increase in the penetration depth $l_{\perp}(\varphi)$. For ${ }^{56} \mathrm{Fe}$ and $14.4-\mathrm{keV}$ radiation, $\varphi_{c}=3.8$ $\mathrm{mrad}$, and for $\varphi=3.0 \mathrm{mrad},|R|^{2} \simeq 0.9$ and $l_{\perp} \simeq 29 \AA$, while at $\varphi=4.5 \mathrm{mrad},|R|^{2} \simeq 0.1$ and $l_{\perp} \simeq 472 \AA$.

For $\varphi>\varphi_{c}$, where deep penetration occurs, it should be possible to strongly suppress the $\mathrm{x}$-ray reflection with an impedance-matched quarter-wave film, just as in the optical coating of lenses. That is, the mirror is coated with a film of proper impedance such that the reflections at the upper and lower interfaces are equal, with the thickness chosen so that the two waves emerge $180^{\circ}$ out of phase. The quarter-wave-film condition gives $l_{\perp}(\varphi)=\frac{1}{4} \lambda\left(\varphi^{2}-\right.$ $\left.\varphi_{c}^{2}\right)^{-1 / 2}$, which is typically $\approx 50-100 \AA$. A quarter-wave film for $1-\AA$ radiation is possible because grazing incidence augments the required film thickness by the factor $\left(\varphi^{2}-\varphi_{c}^{2}\right)^{-1 / 2}$. In addition to impedance-matched quarter-wave GIAR films, we will see that new "damping-stabilized" solutions arise due to the effect of strong photoabsorption.

The fact that $\mathrm{x}$-ray interference between interface re- 
flections can occur at grazing incidence was observed in 1930 by Kiessig ${ }^{16}$ who found half-wave-film interference fringes for grazing-incidence reflection from a film of $\mathrm{Ni}$ on glass. Important extensions in theory and experiment have been made by Alichanow and Arzimowic, ${ }^{17}$ Parratt, ${ }^{18}$ Rieser, ${ }^{19}$ and Segmüller. ${ }^{20}$ As pointed out in these papers, the fringe pattern gives a sensitive measure of film thickness and index of refraction, and offers a probe of surface properties involving variations of electron density with depth. Of course, in these investigations there was no interest in eliminating reflections, so the possibility of antireflection coatings has not been studied.

In this paper we first give a brief review in Sec. II of the theory of grazing-incidence reflection from layered media. In Sec. III we consider the simplest case of a high-density mirror coated with a single lower-density film. We show there are two possible antireflection solutions-impedance-matched $\lambda / 4$ films, and an alternate set of damping stabilized solutions which arise due to photoabsorption. We also examine the effects of longrange film-thickness variations, incident-beam divergence, and the frequency range of suppression.

In the following paper II, ${ }^{21}$ we discuss alternative techniques for suppressing electronic reflections with halfwave films, ultrathin films, tapered impedance films, and general multilayer techniques. In forthcoming papers we examine the following topics. The theory of pure nuclear, coherent $\gamma$-ray reflections using GIAR films is discussed in paper III, 22 and the application to resonant filtering of synchrotron radiation is discussed in paper IV. ${ }^{23}$ In paper $\mathrm{V},{ }^{24}$ we discuss the related idea of resonant superlattice GIAR mirrors which have multiple resonant layers to enhance resonant scattering at larger incidence angles several times $\phi_{c}$. Finally, in paper VI, ${ }^{25}$ we discuss two questions of particular importance for producing highefficiency antireflection films-the effect of surface and volume "roughness" and the limitations imposed by "noise" arising from various sources of incoherent scattering.

\section{REFLECTION FROM LAYERED MEDIA}

Grazing-incidence reflection of $\mathrm{x}$ rays from layered media was first given a general treatment by Parratt. ${ }^{18}$ Also, except for the effects of strong x-ray photoabsorption, the mathematical problem is the same as for reflection of optical radiation from layered dielectric films. ${ }^{1-6}$

\section{A. Grazing incidence reflection}

As was first pointed out by Compton, ${ }^{15}$ critical reflection of $x$ rays is possible due to the small index-ofrefraction decrease in going to a medium of higher electron density. The same results, from a different viewpoint, can be derived directly from the dynamical theory of $\mathrm{x}$-ray and $\gamma$-ray optics. ${ }^{26-28}$ Here we take the index-of-refraction approach. The dynamical development, which is particularly convenient for treating the effects of surface irregularity, is discussed in Appendix A.

For $x$-ray radiation incident near grazing incidence on a flat surface as shown in Fig. 1(a), translational invariance implies that the $(x, y)$ components of the reflected and (a)

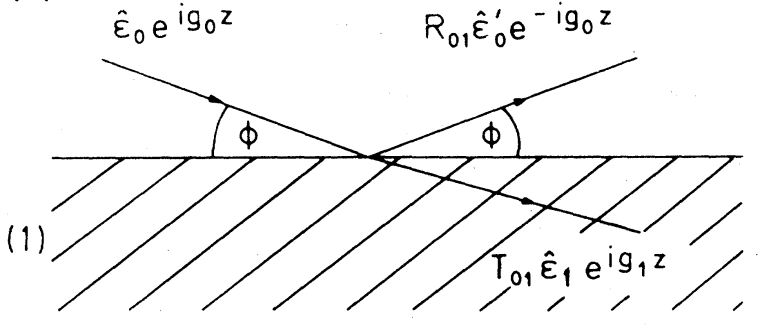

(b)

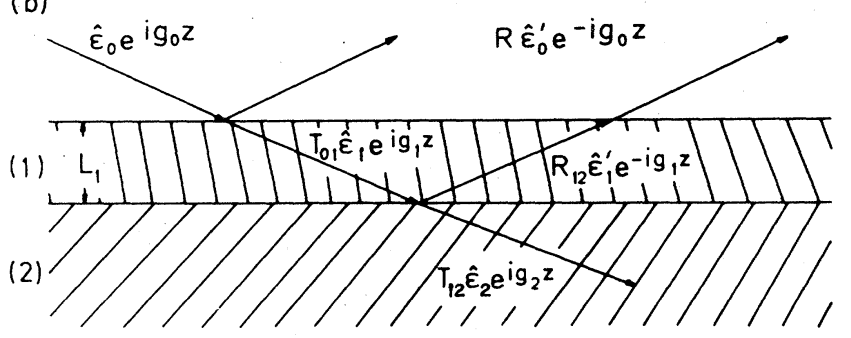

FIG. 1. Grazing-incidence geometry.

transmitted wave vectors must remain unchanged. The incident wave is $\hat{\epsilon}_{0} \exp \left(i g_{0} z\right)$, where

$$
g_{0}=k_{0} \phi
$$

is the $z$ component of the incident wave vector; the reflected wave is $R_{01} \hat{\epsilon}_{0}^{\prime} \exp \left(-i g_{0} z\right)$ and the transmitted wave in medium 1 is $T_{01} \hat{\epsilon}_{1} \exp \left(i g_{1} z\right)$. For nonresonant scattering it is convenient to use the linear $\sigma$ - and $\pi$ polarization basis. It is assumed that the medium is not optically active, so there is no orthogonal scattering $\hat{\boldsymbol{\epsilon}}_{\pi} \rightarrow \hat{\boldsymbol{\epsilon}}_{\sigma}, \hat{\boldsymbol{\epsilon}}_{\sigma} \rightarrow \hat{\boldsymbol{\epsilon}}_{\pi}$. This is normally valid for X-ray scattering but is not valid for resonant $\gamma$-ray optics, which has very strong Faraday-type effects as discussed in paper III. $^{22}$

$$
g_{1} \equiv g_{1}^{\prime}+i g_{1}^{\prime \prime}=k_{0} \phi \beta_{1},
$$

where the "refraction factor" is

$$
\begin{aligned}
\beta_{1} \equiv \beta_{1}^{\prime}+i \beta_{1}^{\prime \prime} & =\left[1+\left(n \lambda^{2} f_{e} / \pi \phi^{2}\right)\right]^{1 / 2} \\
& =\left[1-\left(\phi_{c} / \phi\right)^{2}+i\left(\lambda / \phi^{2} l_{A}\right)\right]^{1 / 2} .
\end{aligned}
$$

Here, $n$ is the atomic density (number of atoms $/ \mathrm{cm}^{3}$ ) and $f_{e}$ is the atomic forward scattering amplitude due to electronic scattering, ${ }^{29}$

$$
f_{e}=-\left(Z+\Delta f^{\prime}\right) r_{0}+i\left(\sigma_{e} / 4 \pi \lambda\right) .
$$

The first term, $Z r_{0}$, where $Z$ is the atomic number and $r_{0}=e^{2} / m c^{2}=2.8 \times 10^{-13} \mathrm{~cm}$ is the classical electron radius, is due to Thomson scattering and gives the dominant real contribution. The second term, $\Delta f^{\prime} r_{0}$, is the anomalous scattering contribution, which is only appreciable near or below an absorption edge. Finally, the imaginary contribution gives the effects of absorption. Here, $\sigma_{e}$ is the total cross section which in the $10-\mathrm{keV} \mathrm{x}$-ray region is dominated by photoabsorption. In the second line of Eq. (3), $\phi_{c}$ is the critical angle, 


$$
\phi_{c}=\left[n \lambda^{2} r_{0}\left(Z+\Delta f^{\prime}\right) / \pi\right]^{1 / 2},
$$

and $l_{A}$ is the absorption length,

$$
l_{A}=\left(n \sigma_{e}\right)^{-1} \text {. }
$$

For a medium with several atomic species, $n f_{e} \rightarrow \sum n_{j} f_{j}$.

The perpendicular penetration depth of the wave in medium 1 is determined by the imaginary part of $g_{1}$,

$$
l_{\perp}(\phi)=\left(2 g_{1}^{\prime \prime}\right)^{-1}=\lambda / 2 \phi \beta_{1}^{\prime \prime} .
$$

Applying the boundary conditions on $E$ and $B$ across the surface gives the Fresnel formula for the reflection amplitude, ${ }^{29}$

$$
\begin{aligned}
R_{01}(\phi) & =\left(\frac{1-\beta_{1}}{1+\beta_{1}}\right) \\
& =\frac{1-\left[1-\left(\phi_{c} / \phi\right)^{2}+i\left(\lambda / \phi^{2} l_{A}\right)\right]^{1 / 2}}{1+\left[1-\left(\phi_{c} / \phi\right)^{2}+i\left(\lambda / \phi^{2} l_{A}\right)\right]^{1 / 2}} .
\end{aligned}
$$

Our primary interest is in Mössbauer $\gamma$ rays and we shall use the $14.4-\mathrm{keV} \gamma$ ray of ${ }^{57} \mathrm{Fe}$ as our main example. In Fig. 2 we plot the reflectivity $\left|R_{01}(\phi)\right|^{2}$ and the penetration depth $l_{\perp}(\phi)$ vs $\phi$ for grazing incidence of $14.4-\mathrm{keV}$ radiation from a flat ${ }^{56} \mathrm{Fe}$ surface. Here, $Z=26$, $\Delta f^{\prime}=0.27$, and $n=8.5 \times 10^{22} / \mathrm{cm}^{3}$, giving a critical angle of $\phi_{c}=3.8 \times 10^{-3} \mathrm{rad}$, and the photoelectric absorption

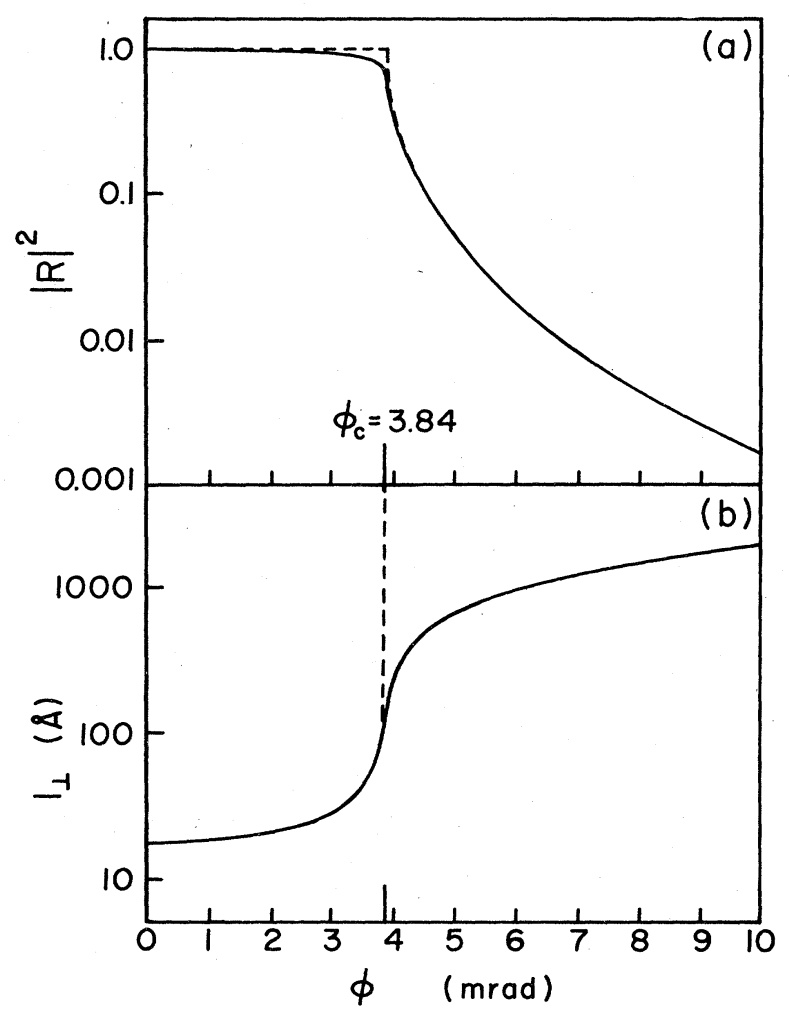

FIG. 2. (a) Reflectivity $|R(\phi)|^{2}$ vs $\phi$ and (b) perpendicular penetration depth $l_{\perp}(\phi)$ vs $\phi$ for grazing-incidence reflection of $0.86-\AA$ radiation from ${ }^{56} \mathrm{Fe}$ (solid curves). The dashed curves give the hypothetical results in the absence of absorption, $\sigma_{e} \rightarrow 0$. cross section is $\sigma_{e}=6.1 \times 10^{-21} \mathrm{~cm}^{2}$, giving a photoabsorption length $l_{A}=1.9 \times 10^{-3} \mathrm{~cm}$.

In the absence of photoabsorption, the reflectivity and penetration depth are shown by the dashed curves in Fig. 2. For $\phi<\phi_{c}, \beta_{1}$ is purely imaginary, $\beta_{1}$ $=\left[1-\left(\phi_{c} / \phi\right)^{2}\right]^{1 / 2}=i\left[\left(\phi_{c} / \phi\right)^{2}-1\right]^{1 / 2}$, and total reflection occurs, $\left|R_{01}\left(\phi<\phi_{c}\right)\right|^{2}=1$. The penetration depth $l_{\perp}(\phi)=\pi / 2\left(\phi_{c}^{2}-\phi^{2}\right)^{1 / 2}$, which is very shallow, $\approx 20 \AA$ over most of the region, but becomes infinite as $\phi \rightarrow \phi_{c}$. For $\phi>\phi_{c}, \beta_{1}$ is real, the reflectivity falls off asymptotically as $\left(\phi_{c} / \phi\right)^{4} / 16 \propto \phi^{-4}$, and the penetration depth is infinite.

The effect of photoabsorption is to decrease the reflectivity, and to limit the penetration depth. Now, as $\phi \rightarrow \phi_{c}$, $l_{\perp} \rightarrow\left(\lambda l_{A} / 2\right)^{1 / 2}=116 \AA$, while for $\phi \gg \phi_{c}, l_{\perp} \approx l_{A} \phi$ $\approx 2.0 \phi \times 10^{5} \AA$, which is the usual penetration depth limited by photoabsorption. Although the penetration depth is now limited, $l_{\perp}$ still becomes very deep for $\phi>\phi_{c}$ increasing from $\approx 20 \AA$ for $\phi<\phi_{c}=3.8 \mathrm{mrad}$, to $116 \AA$ at $\phi=\phi_{c}$, to $472 \AA$ at $\phi=4.5 \mathrm{mrad}$. This rapid increase in $l_{\perp}$ for $\phi>\phi_{c}$ is necessary for antireflection coatings since the penetration depth must exceed the film thickness.

\section{B. Uniform film coating}

For grazing-incidence reflection from a surface coated with a uniform film of thickness $l_{1}$, there are transmitted and reflected waves $\exp \left( \pm i g_{1} z\right)$ in the film and a transmitted wave $\exp \left(\mathrm{ig}_{2} z\right)$ in medium 2, as indicated in Fig. 1(b). Here the complex wave vector $g_{j}=g_{j}^{\prime}+i g_{j}^{\prime \prime}=k_{0} \phi \beta_{j}$. Applying the boundary conditions at the two interfaces gives the reflection amplitude ${ }^{18}$

$$
R(\phi)=\frac{R_{01}+R_{12} e^{i 2 g_{1} l_{1}}}{1+R_{01} R_{12} e^{i 2 g_{1} l_{1}}},
$$

where $R_{01}$ and $R_{12}$ are the reflection amplitudes at the medium interfaces $0-1$ and 1-2, respectively,

$$
\begin{aligned}
& R_{01}(\phi)=\left(1-\beta_{1}\right) /\left(1+\beta_{1}\right), \\
& R_{12}(\phi)=\left(\beta_{1}-\beta_{2}\right) /\left(\beta_{1}+\beta_{2}\right),
\end{aligned}
$$

where for medium $j$,

$$
\begin{aligned}
\beta_{j} & \equiv \beta_{j}^{\prime}+i \beta_{j}^{\prime \prime}=\left\{1+\left[n(j) \lambda^{2} f_{e}(j) / \pi \phi^{2}\right]\right\}^{1 / 2} \\
& =\left\{1-\left[\phi_{c}(j) / \phi\right]^{2}+i\left[\lambda / \phi^{2} l_{A}(j)\right]\right\}^{1 / 2} .
\end{aligned}
$$

\section{Multiple layers}

For a multilayer media, the reflection amplitude can be obtained by iteration: For a substrate covered with two uniform films, the reflection amplitude is

$$
R(\phi)=\frac{R_{01}+e^{i 2 g_{1} l_{1}} R_{12}^{\dagger}}{1+R_{01} R_{12}^{\dagger} e^{i 2 g_{1} l_{1}}},
$$

where $R_{12}^{\dagger}$ is the compound reflectivity arising at the 1-2 interface (from both film 2 and medium 3 ), which is given by Eq. (9): 


$$
R_{12}^{\dagger}=\frac{R_{12}+R_{23} e^{i 2 g_{2} l_{2}}}{1+R_{12} R_{23} e^{i 2 g_{2} l_{2}}}
$$

This iteration procedure can be extended to an arbitrary number of layers, replacing $R_{23}$ by the compound reflectivity $R_{23}^{\dagger}$ in Eq. (13), etc.

An alternative solution can be given in terms of the "characteristic matrices" $\mathscr{M}_{n}$ which relate the $E$ and $B$ fields at the upper and lower boundaries of the $n$th film. This is the customary approach used in treating optical reflections from dielectric thin films ${ }^{1}$ and the formalism can be taken over directly with minor modifications for the strong $x$-ray absorption. This approach is summarized in Appendix B.

\section{IMPEDANCE-MATCHED $\lambda / 4$ FILMS AND DAMPING-STABILIZED SOLUTIONS}

For an antireflection coating, the wave reflected from the interfaces must be of equal amplitude and emerge $180^{\circ}$ out of phase. In this paper we examine the conditions for an antireflection coating when the reflecting mirror is coated with a single film of lower electron density. In the limit of negligible photoabsorption, antireflection coatings for this case are impedance-matched "quarter-wave" films. When photoabsorption is taken into account, the quarter-wave film conditions are modified and, in addition, there are new "damping-stabilized" solutions which are intermediate between $\lambda / 4$ and $\lambda / 2$ films.

\section{A. Limit $\sigma_{e} \rightarrow 0$}

For a mirror (2) coated with a single film (1), the reflection amplitude is given by Eq. (9). Assuming the film has lower electron density, then $\phi_{c 1}<\phi_{c 2}$, and in the region $\phi>\phi_{c 2}, R_{01}, R_{12}$, and $g_{1}$ are purely real when $\sigma_{e}(1)=\sigma_{e}(2)=0$.

The impedance-match condition $R_{01}=R_{12}$ reduces to $\beta_{1}^{2}=\beta_{2}$, which requires an electron density

$$
\begin{aligned}
\rho_{1}(\phi) & \equiv n_{1}\left(Z+\Delta f_{1}^{\prime}\right) \\
& =\rho_{2}\left(\phi / \phi_{c 2}\right)^{2}\left\{1-\left[1-\left(\phi_{c 2} / \phi\right)^{2}\right]^{1 / 2}\right\},
\end{aligned}
$$

and the destructive interference condition $\exp \left(i 2 g_{1} l_{1}\right)$ $=-1$ requires a quarter-wave film: the film thickness should be an odd multiple of

$$
l_{1}(\phi)=\lambda / 4\left(\phi^{2}-\phi_{c 1}^{2}\right)^{1 / 2},
$$

which, using Eq. (14) to determine $\phi_{c 1}$, gives

$$
l_{1}(\phi)=\lambda / 4 \phi\left[1-\left(\phi_{c 2} / \phi\right)^{2}\right]^{1 / 4}
$$

for an impedance-matched quarter-wave film.

Both the required electron density and proper film thickness depend on the angle of incidence $\phi$. An examination of Eqs. (14) and (15) shows that the required electron density varies from $\rho_{1}(\phi)=\rho_{2}$ at $\phi=\phi_{c 2}$ decreasing to $\approx \frac{1}{2} \rho_{2}$ for $\phi \gg \phi_{c 2}$, and the required $l_{1}$ decreases from $\infty$ at $\phi=\phi_{c 2}$ to $\lambda / 4 \phi$ for $\phi \gg \phi_{c 2}$. This behavior is shown explicitly in Figs. 3(a) and 3(b) for the case of 14.4-keV radiation incident on a coated $\mathrm{Fe}$ mirror. Here,

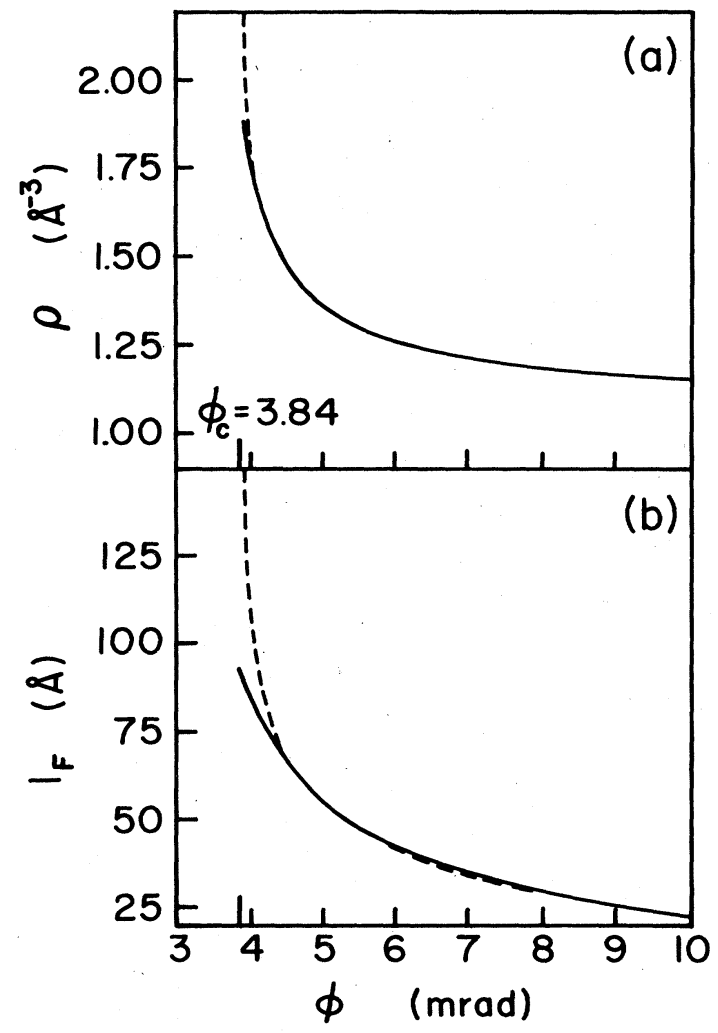

FIG. 3. (a) Required electron density $\rho_{1}(\phi)$ for an impedance-matched film on an ${ }^{56} \mathrm{Fe}$ mirror reflecting $0.86-\AA$ radiation. The dashed curve is for the hypothetical limit $\sigma_{e} \rightarrow 0$, while the solid curve includes photoabsorption. (b) Required film thickness $l_{1}(\phi)$ for an impedance-matched quarter-wave film.

$\rho_{2}=2.24 / \AA^{3}$ and $\phi_{c 2}=3.8 \mathrm{mrad}$, and we see that the required thickness decreases from $\approx 87 \AA$ at $\phi=4.0 \mathrm{mrad}$ to $\approx 25 \AA$ at $\phi=10 \mathrm{mrad}$.

For a coating of given $\rho_{1}$, Eq. (14) determines the angle $\phi_{0}$ at which an impedance match occurs,

$$
\phi_{0}=\left(\rho_{1} / \rho_{2}\right)^{1 / 2} \phi_{c 2} /\left(2-\rho_{2} / \rho_{1}\right)^{1 / 2},
$$

and for a quarter-wave film, the thickness should be taken as $l_{1}\left(\phi_{0}\right)$ as determined by Eq. (15).

For an ${ }^{56} \mathrm{Fe}$ mirror $\left(\rho_{2}=2.24 \AA^{-3}\right)$, there are many possible coatings with $\frac{1}{2} \rho_{2}<\rho_{1}<\rho_{2}$. Table I gives the op-

TABLE I. Optimal parameters $l_{1}, \phi_{0}$ for several coatings on $\mathrm{Fe}$, reflecting 14.4-keV radiation in the limit $\sigma_{e} \rightarrow 0$.

\begin{tabular}{lccc}
\hline \hline & $\rho_{1} / \rho_{2}$ & $\begin{array}{c}\phi_{0} \\
(\mathrm{mrad})\end{array}$ & $\begin{array}{c}l_{1} \\
(\AA)\end{array}$ \\
\hline $\mathrm{Ti}$ & 0.55 & 6.30 & 38.4 \\
$\mathrm{Se}$ & 0.56 & 6.26 & 38.7 \\
$\mathrm{Ge}$ & 0.64 & 4.68 & 60.9 \\
$\mathrm{Te}$ & 0.69 & 4.31 & 74.4 \\
$\mathrm{Ga}$ & 0.71 & 4.21 & 80.3 \\
$\mathrm{~V}$ & 0.74 & 4.14 & 85.2 \\
$\mathrm{Sb}$ & 0.76 & 4.05 & 95.2 \\
$\mathrm{Ce}$ & 0.76 & 4.04 & 96.6 \\
$\mathrm{Zr}$ & 0.77 & 4.02 & 99.6 \\
\hline \hline
\end{tabular}




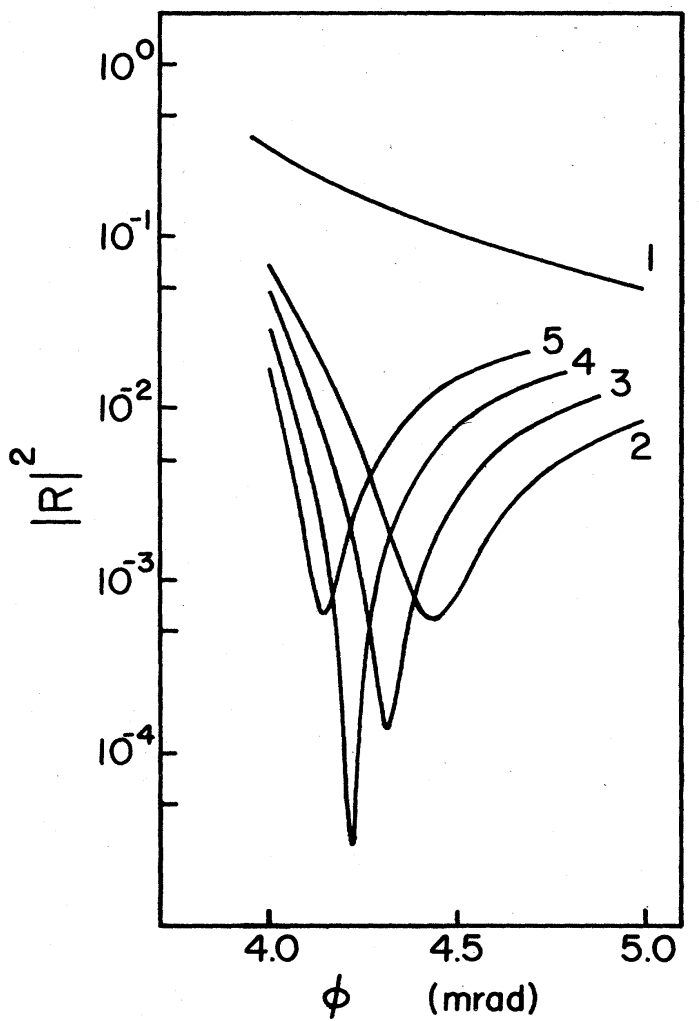

FIG. 4. $|R|^{2}$ vs $\phi$ for ${ }^{56} \mathrm{Fe}$ coated with (1) uncoated, (2) 70$\AA$ Te, (3) 75- $\AA \mathrm{Te},(4) 80-\AA \mathrm{Te}$, and (5) $85-\AA \mathrm{A} \mathrm{Te}$.

timum parameters $\phi_{0}, l_{1}\left(\phi_{0}\right)$ determined from Eqs. (15) and (16) for several possible impedance-matched quarterwave films on $\mathrm{Fe}$ for $14.4-\mathrm{keV}$ radiation. In these cases the optimal parameters range from $l_{1}=38 \AA$ 作 $\phi_{0}=6.3$ $\operatorname{mrad}$ for $\mathrm{Ti}$ to $l_{1}=100 \AA, \phi_{0}=4.0 \mathrm{mrad}$ for the higherdensity $\mathrm{Zr}$ coat.

Figure 4 shows rocking curves $|R|^{2}$ vs $\phi$ for different thicknesses of $\mathrm{Te}$ on $\mathrm{Fe}$, where, here, photoabsorption is taken into account. We note the position $\phi_{\min }$ of minimum reflectivity changes with thickness due to the changing quarter-wave condition. There is an optimal thickness $l_{1} \approx 80 \AA$ and an optimal angle of incidence $\phi_{0} \approx 4.2 \mathrm{mrad}$ for maximum suppression. These optimal parameters are close to the estimate of Eqs. (15) and (16) given in Table I but are appreciably shifted by the effects of photoabsorption. Of course, the most interesting feature of Fig. 4 is that very significant suppression occurs, with the reflectivity reduced by $3-4$ orders of magnitude.

\section{B. General treatment for $\sigma_{e} \neq 0$}

In the limit $\sigma_{e} \rightarrow 0$ discussed above, the antireflection coating is restricted to an electron density $\frac{1}{2} \rho_{2} \leq \rho_{1} \leq \rho_{2}$ and reflection must occur in the region $\phi>\phi_{c 2}\left(>\phi_{c 1}\right)$. For $\sigma_{e} \neq 0$, there are significant shifts in the optimum parameters $\phi_{0}, l_{1}$ for a quarter-wave-film antireflection coating, and there is a new set of damping-stabilized solutions for antireflection coatings in the previously inaccessible region $\phi_{c 1}<\phi<\phi_{c 2}$, which also allow low-density coatings $\rho_{1}<\frac{1}{2} \rho_{2}$

To treat the general case, we rewrite the reflection amplitude Eq. (9) as

$$
R(\phi)=\frac{r_{01} e^{i \phi_{01}}+r_{12} e^{-2 g_{1}^{\prime \prime} l_{1}} e^{i\left(\phi_{12}+2 g_{1}^{\prime} l_{1}\right)}}{1+R_{01} R_{12} e^{i 2 g_{1} l_{1}}},
$$

where we have introduced the amplitudes and phases of the interface reflection amplitudes,

$$
\begin{aligned}
& R_{01}(\phi)=r_{01}(\phi) e^{i \phi_{01}(\phi)}, \\
& R_{12}(\phi)=r_{12}(\phi) e^{i \phi_{12}(\phi)},
\end{aligned}
$$

and, as before, $g_{1}^{\prime}$ and $g_{1}^{\prime \prime}$ are the real and imaginary parts of the complex wave vector.

The behavior of the separate interface reflection amplitudes and phases are shown in Fig. 5 for $\sigma_{e}=0$ and in Fig. 6 for $\sigma_{e} \neq 0$, again for Te on Fe reflecting 14.4-keV radiation: In Fig. 5(a) the solid curve gives $r_{01}(\phi)$, the magnitude of the reflection amplitude from the top film surface, while the dotted curve gives $r_{12}(\phi)$ $\times \exp \left[-2 g_{1}^{\prime \prime}(\phi) l_{1}\right]$, the magnitude of the wave amplitude reflected from the film-mirror interface, including the extinction effects of propagating through the film. Since here $\sigma_{e}=0$, then $g_{1}^{\prime \prime}(\phi)=0$ for $\phi>\phi_{c 1}$ and in this region the dotted curve coincides with $r_{12}(\phi)$. However, for $\phi<\phi_{c 1}$, there is strong primary extinction $\left(g_{1}^{\prime \prime} \neq 0\right)$, so the reflected amplitude depends on the film thickness $l_{1}$ and is strongly reduced from $r_{12}$. [In the region $\phi<\phi_{c 1}$, the dashed curve gives $r_{12}(\phi)$.] Now, $r_{01}(\phi)$ is just the usual grazing-incidence reflection amplitude from a single surface and exhibits the features discussed in Sec. II A: Total reflection occurs for $\phi<\phi_{c 1}$ (where $\beta_{1}$ is imaginary), rapidly decreasing $\sim \phi_{c 1}^{2} / 4 \phi^{2}$ for $\phi>\phi_{c 1}$ (where $\beta_{1}$ is real). On the other hand, $r_{12}(\phi)$ only exhibits total reflection in

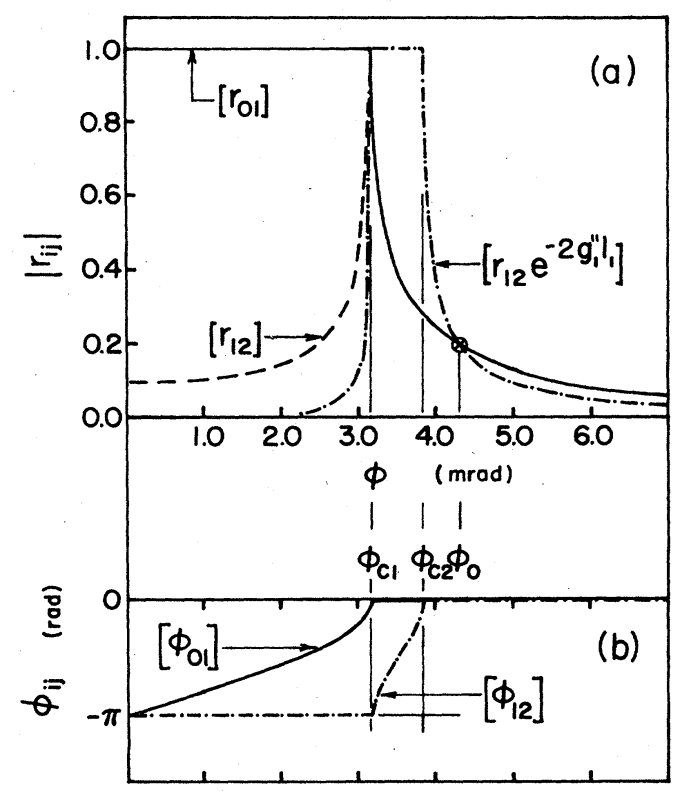

FIG. 5. (a) Interface reflection amplitudes vs $\phi$ and (b) phases of the interface reflections vs $\phi$ for $80-\AA \mathrm{Te}$ on $\mathrm{Fe}\left(\sigma_{e}=0\right)$. 
the region $\phi_{c 1} \leq \phi \leq \phi_{c 2}$ (where $\beta_{1}$ is real and $\beta_{2}$ imaginary) and falls off rapidly $\sim\left(\phi_{c 2}^{2}-\phi_{c 1}^{2}\right) / 4 \phi^{2}$ for $\phi>\phi_{c 2}$ (where both $\beta_{1}$ and $\beta_{2}$ are real) and for $\phi<\phi_{c 1}$ (where both $\beta_{1}$ and $\beta_{2}$ are imaginary). In the latter region,

$$
r_{12}(\phi) \rightarrow\left(\phi_{c 2}-\phi_{c 1}\right) /\left(\phi_{c 2}+\phi_{c 1}\right)
$$

as $\phi \rightarrow 0$.

Figure 6(a) includes the effects of photoabsorption. The solid curve again gives $r_{01}(\phi)$ and the dotted curve gives $r_{12}(\phi) \exp \left(-2 g_{1}^{\prime \prime} l_{1}\right)$. However, now $g_{1}^{\prime \prime} \neq 0$ for all $\phi$, so the effective reflection amplitude of the film-mirror interface depends explicitly on the film thickness for all $\phi$. Here the curves are calculated for $80 \AA$ of $\mathrm{Te}$ on ${ }^{56} \mathrm{Fe}$. The primary effect of photoabsorption is to reduce the reflection amplitudes somewhat and produces a general "degradation" of the curves.

Figures $5(\mathrm{~b})$ and $6(\mathrm{~b})$ give the phases of the reflection amplitudes for $\sigma_{e}=0$ and $\sigma_{e} \neq 0$, respectively. In both cases, the solid curve gives $\phi_{01}(\phi)$ (the phase of the reflection from the 0-1 interface) and the dashed curve gives $\phi_{12}(\phi)$ (the phase of the 1-2 interface reflection). We see in Fig. 5(b) that in the absence of photoabsorption, the two phases vary rapidly in the corresponding totalreflection regions of $r_{01}$ and $r_{12}$, with $\phi_{01}$ increasing from $-\pi \rightarrow 0$ as $\phi \propto 0 \rightarrow \phi_{c 1}$ and with $\phi_{12}$ increasing from $-\pi \rightarrow 0$ as $\phi \propto \phi_{c 1} \rightarrow \phi_{c 2}$, and that outside these regions, the phases remain constant. Again photoabsorption gives a small degradation of the curves as shown in Fig. 6(b).

The rapid variation of the phases is reminiscent of the rapid phase variation in the region of total Bragg reflection. This of course is not coincidental: From the point of view of the dynamical theory, ${ }^{26,27}$ grazing-incidence reflection from a single surface can be viewed as a zeroangle Bragg reflection, with the region of total Bragg reflection being $0 \leq \phi \leq \phi_{c}$. The dynamical treatment is dis-

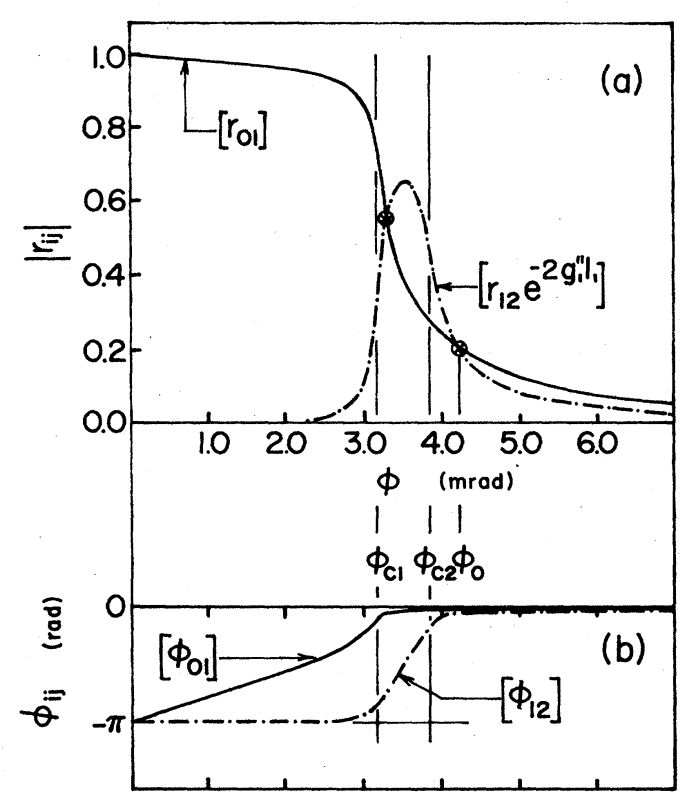

FIG. 6. Interface reflection amplitudes and phases vs $\phi$ for $80-\AA \mathrm{Te}$ on $\mathrm{Fe}$ at $14.4 \mathrm{keV}\left(\sigma_{e} \neq 0\right)$. cussed in Appendix A. We now return to the total reflection amplitude of Eq. (17) and examine the general conditions for an antireflection coating.

\section{Impedance match}

The general impedance-match condition is

$$
r_{01}(\phi)=r_{12}(\phi) e^{-2 g_{1}^{\prime \prime}(\phi) l_{1}} \text {. }
$$

In the limit of no photoabsorption, $g_{1}^{\prime \prime}(\phi)=0$ (for $\phi>\phi_{c 1}$ ) and the impedance-match condition then determines a unique electron density $\rho_{1}(\phi)$, as given by Eq. (14), which produces an impedance match at the angle $\phi$. Alternatively, the impedance-match condition determines the angle $\phi_{0}$ at which exact impedance match occurs. In Fig. 5(a) $\phi_{0}$ is determined by the intersection of the curves for $r_{01}(\phi)$ and $r_{12}(\phi)$ in the region $\phi>\phi_{2}$. An intersection for impedance match also occurs at $\phi_{0}^{\prime}=\phi_{c 1}$, where total reflection occurs at both interfaces. In this limit $\left(\sigma_{e}=0\right)$, the intersections $\phi_{0}, \phi_{0}^{\prime}$ are independent of the film thickness $l_{1}$.

However, when photoabsorption is included, the impedance-match condition, Eq. (19), depends on the film thickness $l_{1}$ as well as $\phi$, which has the following effect: For a given $l_{1}$, if an exact match occurs at the angle $\phi_{0}$, i.e.,

$$
r_{01}\left(\phi_{0}\right)=r_{12}\left(\phi_{0}\right) \exp \left[-2 g_{1}^{\prime \prime}\left(\phi_{0}\right) l_{1}\right],
$$

a change in film thickness will cause a mismatch. Thus, photoabsorption causes the impedance-match angle $\phi_{0}$ to be dependent on $l_{1}$ and now, rather than a single $\phi_{0}$ (with arbitrary thickness), there is a region of impedance-match angles $\phi_{0}\left(l_{1}\right)$ and a range of film thickness $l_{1}$ for which it is possible to achieve an impedance match.

Figure 7(a) gives the curves for $r_{01}(\phi)$ and $r_{12}(\phi) \exp \left(-2 g_{1}^{\prime \prime} l_{1}\right)$ for various film thickness $l_{1}$ (Te on $\left.\mathrm{Fe}, E_{\gamma}=14.4 \mathrm{keV}\right)$. From these curves, it is clear that due to photoextinction of the lower reflection there is a maximum thickness $l_{1}^{*}$ beyond which no impedance match is possible. For any $l_{1}<l_{1}^{*}$, there are two intersections: $\phi_{0}\left(l_{1}\right)$ which lies in the region $\phi_{c 2} \lesssim \phi_{0}\left(l_{1}\right)<\phi_{0}$ and decreases with increasing $l_{1}$, and $\phi_{0}^{\prime}\left(l_{1}\right)$ which lies in the region $\phi_{c 1}<\phi_{0}^{\prime}\left(l_{1}\right) \leqslant \phi_{c 2}$ and increases with increasing $l_{1}$.

From the impedance-match condition, Eq. (19), we can solve for $l_{1}^{(=)}(\phi)$, the film thickness which gives an exact impedance match at the angle $\phi$,

$$
l_{1}^{(=)}(\phi)=l_{\perp}^{(1)}(\phi) \ln \left[r_{12}(\phi) / r_{01}(\phi)\right],
$$

where, as defined in Eq. (7), $l_{1}^{(1)}(\phi)=\left[2 g_{1}^{\prime \prime}(\phi)\right]^{-1}$ gives the maximum perpendicular penetration depth permitted in the film medium for a wave incident at the angle $\phi$. We note that Eq. (20) requires $r_{12}>r_{01}$, i.e., the reflection at the second interface must be stronger than the reflection from the first interface, which of course is necessary to offset the absorption in propagating through film 1.

The solid line in Fig. $7(\mathrm{~b})$ gives $l_{1}^{(=)}(\phi)$ for $\mathrm{Te}$ on $\mathrm{Fe}$ and $E_{\gamma}=14.4 \mathrm{keV}$. For this case it is possible to find film thicknesses satisfying the impedance-match condition in the region $\phi_{c 1}=3.1 \mathrm{mrad}$ to $\phi_{0}=4.3 \mathrm{mrad}$, with the maximum possible thickness being $l_{1}^{*}=490 \AA$ at 


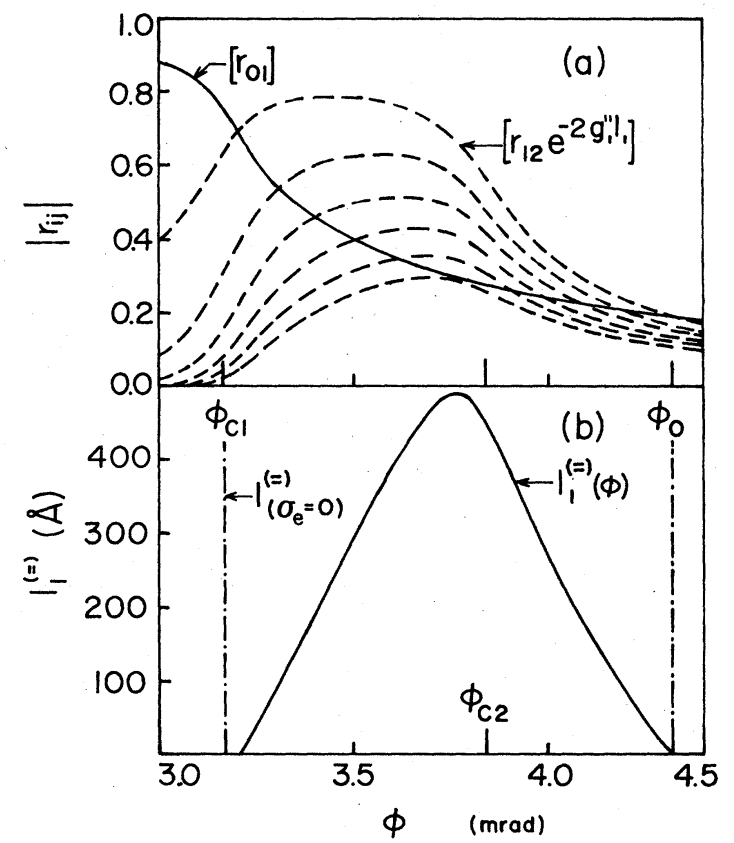

FIG. 7. (a) Lower interface reflection amplitude $r_{12}(\phi) \exp \left[-2 g_{1}^{\prime \prime}(\phi) l_{1}\right]$ vs $\phi$ for $l_{1}=0,100, \ldots, 500 \AA$ (dashed curves) and upper interface $r_{01}(\phi)$ vs $\phi$ (solid curve) for Te on Fe at $14.4 \mathrm{keV}$. (b) Required film thickness $l_{1}{ }^{(=)}(\phi)$ for exact impedance match at angle $\phi$. Dashed line gives limiting case of $\sigma_{e}=0$.

$\phi=3.8 \mathrm{mrad} \simeq \phi_{c 2}$. The dashed line in Fig. 7(b) gives the limiting case of $\sigma_{e}=0$. In this limit, impedance match occurs for any thickness at $\phi_{c 1}$ and at $\phi_{0}$, giving vertical dashed lines for $l_{1}^{(=)}$at these two angles.

\section{Destructive interference}

Returning to Eq. (17), the condition for destructive interference is

$$
\exp \left\{i\left[\phi_{12}(\phi)+2 g_{1}^{\prime}(\phi) l_{1}-\phi_{01}(\phi)\right]\right\}=-1 ;
$$

which requires the phase to be $m \pi, m$ being odd. The corresponding film thickness $l_{1}^{m \pi}(\phi)$ which gives such a relative phase shift to the reflected waves (when the incidence angle is $\phi$ ) is

$$
l_{1}^{m \pi}(\phi)=\left[m \pi-\phi_{12}(\phi)+\phi_{01}(\phi)\right] / 2 g_{1}^{\prime}(\phi) .
$$

The required film thickness decreases with increasing $\phi$ because the phase change only involves the $z$ component of the refracted wave $g_{1}^{\prime}$ which increases with $\phi$ (for $\left.\phi>\phi_{c 1}, \quad\left[2 g_{1}^{\prime}(\phi)\right]^{-1} \simeq \lambda / 4 \pi\left(\phi^{2}-\phi_{c 1}^{2}\right)^{1 / 2}\right)$. Furthermore, for low-density coatings $\left(\rho_{1}<\rho_{2}\right)$ and $\phi>\phi_{c 2}$, $\phi_{12}(\phi)=\phi_{01}(\phi)=0$, as shown in Figs. 5(b) and 6(b), and Eq. (22), gives the quarter-wave-film thickness discussed earlier, $\quad l_{1}^{m \pi} \simeq m\left[\lambda / 4\left(\phi^{2}-\phi_{c 1}^{2}\right)^{1 / 2}\right]$. However, for $\phi_{c 1} \leq \phi \leq \phi_{c 2}, \phi_{12}(\phi)$ varies rapidly from $-\pi$ to 0 , while $\phi_{01}(\phi) \simeq 0$ and the required film thickness is intermediate between a quarter- to a half-wave film.

In Fig. 8 the $l_{1}^{m \pi}(\phi)$ curves are given for $m=1,3,5$,

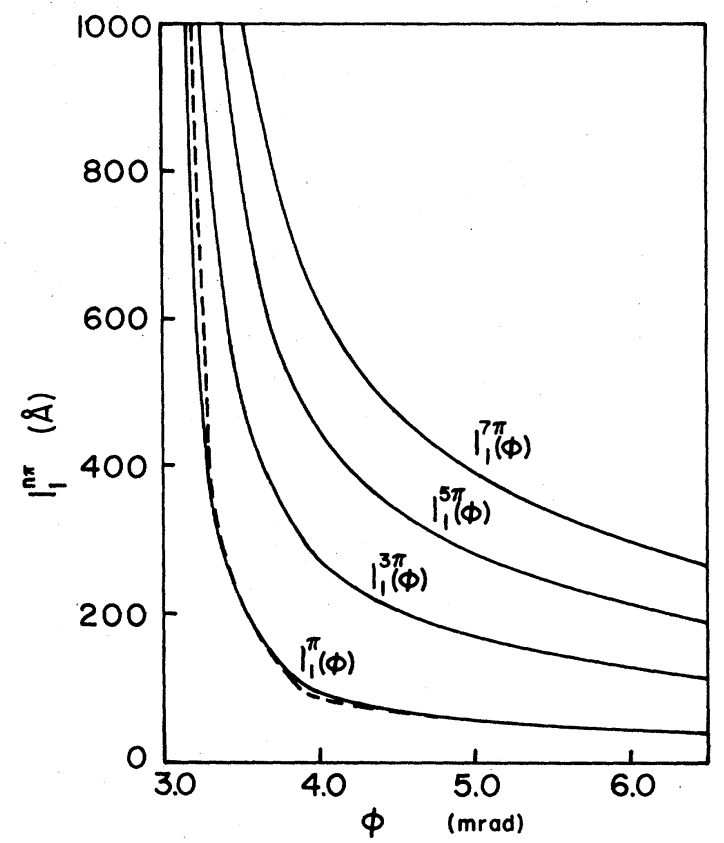

FIG. 8. Required film thickness $l_{1}^{m \pi}(\phi)$ to produce an $m \pi$ relative phase shift of lower reflection at angle $\phi$ for $m=1,3,5$, and 7 , for $\mathrm{Te}$ on $\mathrm{Fe}$ at $14.4 \mathrm{keV}$.

and 7 for $\mathrm{Te}$ on $\mathrm{Fe}$ and $14.4 \mathrm{keV}$. The dashed curve gives the limit $\sigma_{e} \rightarrow 0$, while the solid curves include photoabsorption.

\section{Antireflection coatings}

The intersections of $l_{1}^{(=)}(\phi)$ with the curves $l_{1}^{m \pi}(\phi)$ determine the required film thicknesses and angles of incidence for impedance-matched antireflection coatings which will strongly suppress $x$-ray scattering.

Figure 9(a) shows the limit $\sigma_{e}=0$ for $\mathrm{Te}$ on Fe. Here, $l_{1}^{(=)}(\phi)$ is two vertical lines at $\phi_{c 1}=3.1 \mathrm{mrad}$ and $\phi_{0}=4.3$ mrad, and since all the curves $l_{1}^{m \pi}(\phi) \rightarrow \infty$ as $\phi$ decreases to $\phi_{c 1}$, intersections only occur at $\phi_{0}$. These are simply the quarter-wave films discussed earlier: a GIAR coating requires $73 \AA$ of $\mathrm{Te}$ on $\mathrm{Fe}$ and all other possible coatings are odd multiples of this thickness.

In contrast, when photoabsorption is taken into account as in Fig. 9(b), there are only a finite number of possible coatings, the thickness and operating angles are strongly shifted, and a new set of damping-stabilized solutions appear: Due to photoabsorption, the impedance-match curve $l_{1}^{(=)}(\phi)$ is now deformed from two parallel lines to a bell-shaped curve in the region $\phi_{c 1} \lesssim \phi \lesssim \phi_{0}$, with maximum near $\phi_{c 2}$. The $\pi$-phase curves $l_{1}^{\widetilde{m} \pi}(\widetilde{\phi})$ monotonically decrease in this region and each curve that intersects $l_{1}^{(=)}(\phi)$ will have two intersections-one at $\phi_{0}(m \pi)$ in the region $\phi_{c 2} \lesssim \phi<\phi_{0}$ with thickness $l_{1}(m \pi)$, and one at $\phi_{0}^{\prime}(m \pi)$ in the region $\phi_{c 1}<\phi \lesssim \phi_{c 2}$ with thickness $l_{1}^{\prime}(m \pi)$.

The antireflection coatings $l_{1}(m \pi)$, which have operating angles $\phi_{0}(m \pi)$ in the region $\phi_{c 2} \rightarrow \phi_{0}$, are quarter-wave films, but with significant shifts in the parameters from the $\sigma_{e}=0$ limit. The operating angle $\phi_{0}(m \pi)$ is decreased from $\phi_{0}$ and correspondingly the appropriate thickness 


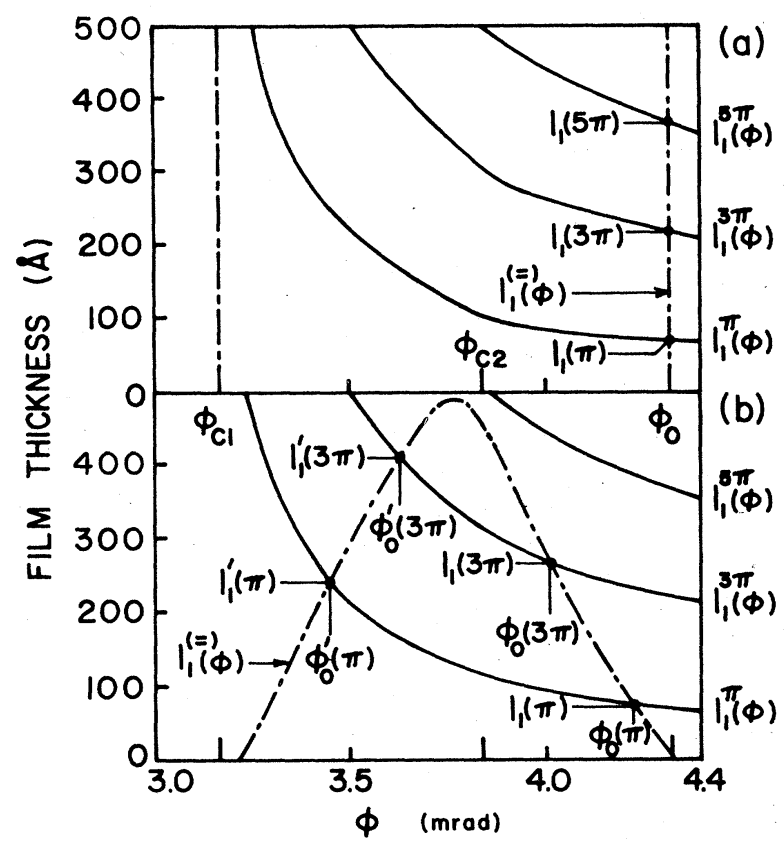

FIG. 9. Graphical solution for the film thickness $l_{1}(m \pi)$ and operating angle $\phi_{0}(m \pi)$ required for an antireflective film. (a) gives the limit $\sigma_{e}=0$. In (b), the new intersections $l_{1}^{\prime}(m \pi), \phi_{0}^{\prime}(m \pi)$ give the damping-stabilized solutions.

$l_{1}(m \pi)$ is increased. Also, $l_{1}(m \pi)$ is no longer a simple multiple of $l_{1}(\pi)$.

The new solutions $l_{1}^{\prime}(m \pi)$, which have operating angles $\phi_{0}^{\prime}(m \pi)$, in the region $\phi_{c 1} \rightarrow \phi_{c 2}$, are only possible due to photoabsorption and will be referred to as dampingstabilized solutions. They have a simple origin: In the absence of photoabsorption there is total reflection from the lower surface 2 for $\phi_{c 1} \leq \phi \leq \phi_{c 2}$ and a weaker reflection from the upper surface 1 , so exact cancellation is never possible. With photoabsorption, extinction occurs in film 1 which reduces the reflected wave from 2 , with the reduction being stronger as $\phi$ decreases to $\phi_{c 1}$ due to the increased path length of the wave in the film. Therefore, as $\phi \rightarrow \phi_{c 1}$, there will always be an angle $\phi_{(=)}^{\prime}$ at which the two reflected waves are equal in magnitude, and by appropriate choice of thickness, phase cancellation will occur.

Because $l_{1}^{m \pi}(\phi)$ monotonically decreases, the dampingstabilized GIAR film will always be thicker than the corresponding $\lambda / 4$ film, i.e., $l_{1}^{\prime}(m \pi)>l_{1}(m \pi)$. Also, because of the rapid variation of the phase $\phi_{12}(\phi)$ in the region $\phi_{c 1} \leq \phi \leq \phi_{c 2}, \quad l_{1}^{\prime}(m \pi)$ will be intermediate between a quarter- to a half-wave film.

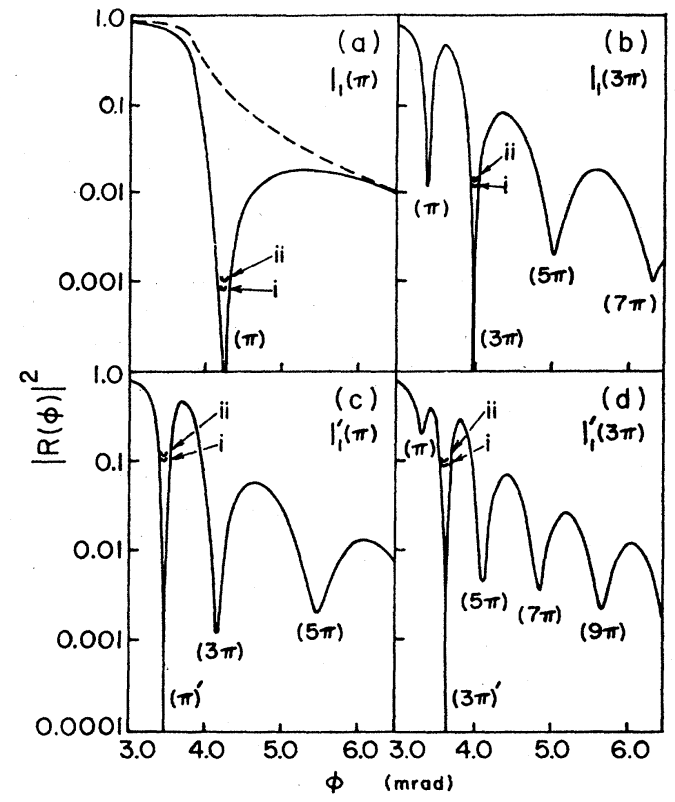

FIG. 10. Rocking curves $|R|^{2}$ vs $\phi$ for reflection of 14.4$\mathrm{keV}$ radiation from the four possible GIAR films of $\mathrm{Te}$ on $\mathrm{Fe}$. The two curves ( $i$ and ii) at the principal minima give the reflectivity averaged over an incident beam spread of $\Delta \phi=0.2 \mathrm{mrad}$ and a double average over beam spread and a long-range film thickness variation $l_{1} \pm 0.025 l_{1}$.

The parameters obtained from Fig. 9 for the possible GIAR films of $\mathrm{Te}$ on $\mathrm{Fe}$ are tabulated in Table II, and Fig. 10 gives the rocking curves $|R(\phi)|^{2}$ vs incidence angle $\phi$ for each of the possible film thicknesses, $l_{1}=l_{1}(m \pi)$ or $l_{1}^{\prime}(m \pi)$. In each case, a very deep minimum occurs at the angle $\phi_{0}(m \pi)$ or $\phi_{0}^{\prime}(m \pi)$ for which an impedancematched antireflection condition holds. The additional minima occur at those angles at which the film gives any odd-order $\pi$-phase shift to the lower reflection, i.e., at $\phi$ such that $l_{1}^{m \pi}(\phi)=l_{1}$, which can be determined graphically from Fig. 8. Although there is no impedance match for these subsidiary minima, they are still quite pronounced, particularly at larger angles $\phi \gtrsim \phi_{0}$, where the difference $\left[r_{01}-r_{12} \exp \left(-2 g_{1}^{\prime \prime} l_{1}\right)\right]$ is small [see Figs. 6(a) and $7(a)]$.

\section{Beam divergence and film-thickness variations}

Two obvious factors limit the degree of suppression: (1) the divergence of the incident beam (or equivalently, the long-range variation of the local surface normal) and (2) the long-range variation of the thickness of the film. The

TABLE II. Parameters for the possible GIAR films of Te on Fe for reflection of 14.4-keV radiation.

\begin{tabular}{|c|c|c|c|c|c|c|}
\hline \multicolumn{2}{|c|}{$\sigma_{e}=0$} & \multicolumn{5}{|c|}{$\sigma_{e} \neq 0$} \\
\hline $\begin{array}{c}\phi_{0}(m \pi) \\
(\mathrm{mrad})\end{array}$ & $\begin{array}{c}l_{1}(m \pi) \\
(\AA \AA \AA)\end{array}$ & $\begin{array}{c}\phi_{0}(m \pi) \\
(\mathrm{mrad})\end{array}$ & $\begin{array}{c}l_{1}(m \pi) \\
(\AA)\end{array}$ & $\begin{array}{c}\phi_{0}^{\prime}(m \pi) \\
(\mathrm{mrad})\end{array}$ & $\begin{array}{c}l_{1}^{\prime}(m \pi) \\
(\AA \AA)\end{array}$ & $m$ \\
\hline 4.32 & 73 & 4.22 & 80 & 3.45 & 240 & 1 \\
\hline 4.32 & 219 & 4.01 & 268 & 3.63 & 409 & 3 \\
\hline 4.32 & 365 & & & & & 5 \\
\hline
\end{tabular}


much more fundamental question of the effect of the microscopic "surface roughness" is discussed in paper VI. ${ }^{25}$

For the synchrotron source DORIS, the vertical divergence of the $\mathrm{x}$-ray beam at $10 \mathrm{keV}$ is $\approx 0.1-0.2 \mathrm{mrad}^{30}$ The long-range thickness control in producing thin films is about $5 \%$ absolute thickness. ${ }^{31}$

For a $\lambda / 4-G I A R$ film $\left[\phi_{0}(m \pi)>\phi_{c 2}\right]$, one main effect of beam divergence and film thickness variations is a change of the quarter-wave condition. Neglecting photoabsorption, if a film of thickness $l_{1}$ is a quarter-wave film for a wave incident at $\phi_{0}$, then the phase change for propagation through the film is

$$
2 l \phi_{0}\left[1-\left(\phi_{c 1} / \phi_{0}\right)^{2}\right]^{1 / 2} / \lambda=m \pi .
$$

For film $l^{\prime}=l+\Delta l$ and a wave incident at $\phi=\phi_{0}+\Delta \phi$,

$$
2 l_{1}^{\prime} \phi\left[1-\left(\phi_{c 1} / \phi\right)^{2}\right]^{1 / 2} \lambda=m \pi+\epsilon,
$$

where $\epsilon=\epsilon(\Delta \phi)+\epsilon(\Delta l)$,

$$
\begin{aligned}
& \epsilon(\Delta \phi)=\frac{\Delta \phi}{\phi_{0}} m \pi \phi_{0}^{2} /\left(\phi_{0}^{2}-\phi_{c 1}^{2}\right), \\
& \epsilon(\Delta l)=\frac{\Delta l}{l_{1}} m \pi .
\end{aligned}
$$

Assuming the film is also impedance matched so that $r_{01}=r_{12}$ at $\phi_{0}$, then at $\phi_{0}+\Delta \phi$ and $l_{1}+\Delta l$,

$$
|R|^{2} \approx\left|R_{01}+R_{12} e^{i 2 l_{1}^{\prime} g_{1}}\right|^{2} \approx\left|r_{01}\right|^{2} \epsilon^{2} .
$$

For a beam divergence of $0.2 \mathrm{mrad}$ and thickness control to $\Delta l / l_{1}= \pm 0.025,|\epsilon| \lesssim 0.2$ and $|R|^{2} \lesssim 0.04\left|r_{01}\right|^{2}$ (for the lowest-order film $m=1$ ). At an angle where the uncoated medium 2 would have $\approx 10 \%$ reflectivity, i.e., $r_{02} \approx 0.3$, the impedance-matched coated medium will have reduced reflectivities at each interface, typically $r_{01} \approx r_{12} \approx 0.15$, so that the coated medium will typically have a reflectivity $|R|^{2} \lesssim 10^{-3}$ over the entire divergence and thickness variation of the film. These effects will be more severe for the higher-order films.

A second effect, which we have neglected above, is the variation of the reflection amplitudes with $\phi$ : Even with an exact impedance match at $\phi_{0}$, i.e., $r_{01}\left(\phi_{0}\right)=r_{12}\left(\phi_{0}\right)$, there will be a mismatch at $\phi_{0}+\Delta \phi$,

$$
r_{01}\left(\phi_{0}+\Delta \phi\right)-r_{12}\left(\phi_{0}+\Delta \phi\right) \simeq\left(d r_{01} / d \phi-d r_{12} / d \phi\right) \Delta \phi .
$$

For $\lambda / 4$-GIAR films $\left[\phi_{0}(m \pi)>\phi_{c 2}\right]$, both amplitudes have negative slope and are already small $(\lesssim 0.1)$, so that the difference remains small over a fairly broad angular region. For the $80-\AA \lambda / 4-G I A R$ film of $\mathrm{Te}$ on $\mathrm{Fe}$, $\left|r_{01}-r_{12} \exp \left(-2 g_{1}^{\prime \prime} l_{1}\right)\right|^{2}$ remains $\leq 10^{-3}$ over an angular region $\Delta \phi=0.46 \mathrm{mrad}$ about $\phi_{0}$, as shown in Fig. 11. This is about the same order effect as that produced by the phase variation and, taken together, the reflectivity will typically be $|R|^{2} \lesssim 10^{-3}$ over a $\Delta \phi \simeq 0.2-0.3 \mathrm{mrad}$.

These estimates are modified for damping-stabilized GIAR film $\left(\phi_{c 1}<\phi_{0}^{\prime}<\phi_{c 2}\right)$ leading to a much more rapid $\Delta \phi$ variation but less sensitivity to $\Delta l$ variations: First, there is now a rapid impedance mismatch with $\Delta \phi$ because $r_{01}$ and $r_{12}$ are larger and have opposite slopes at $\phi_{0}^{\prime}$. [For the example of the 240-A GIAR film of Te on

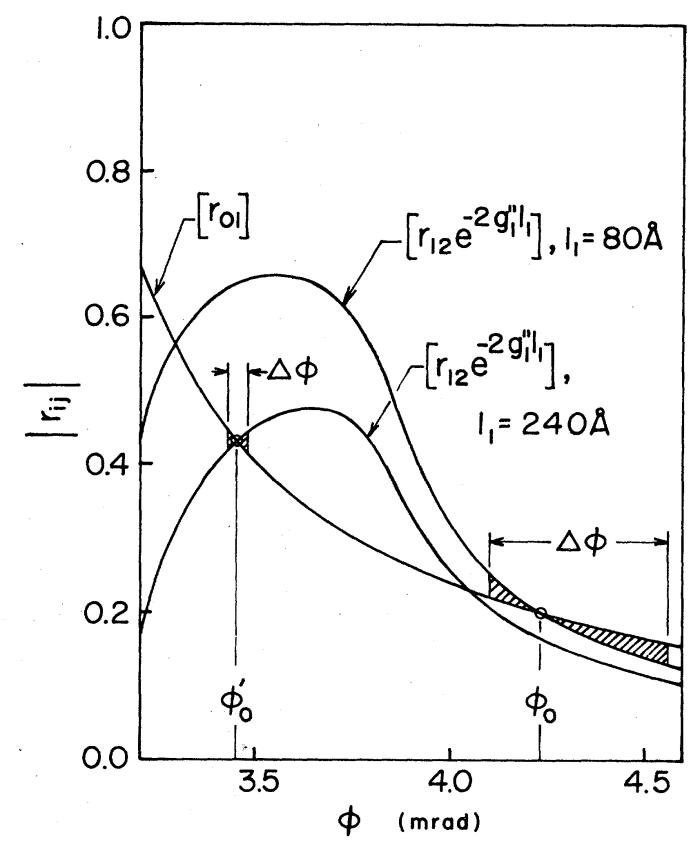

FIG. 11. Interface reflection amplitudes $r_{01}(\phi)$ and $r_{12}(\phi) \exp \left[-2 g_{1}^{\prime \prime}(\phi) l_{1}\right]$ vs $\phi$ for Te films on $\mathrm{Fe}$ at $14.4 \mathrm{keV}$ for $l_{1}=80$ and $240 \AA$. Shaded areas show regions over which $\left|r_{01}-r_{12} \exp \left(-2 g_{1}^{\prime \prime} l_{1}\right)\right|^{2} \leq 10^{-3}$.

Fe in Fig. 11, $\left|r_{01}-r_{12} \exp \left(-2 g_{1}^{\prime \prime} l_{1}\right)\right|^{2} \lesssim 10^{-3}$ only over $\Delta \phi=0.06 \mathrm{mrad}$ about $\phi_{0}^{\prime}$.] Second, the phase change due to film 1 is now

$$
2 l_{1} \phi_{0}^{\prime}\left[1-\left(\phi_{c 1} / \phi_{0}^{\prime}\right)^{2}\right]^{1 / 2} / \lambda+\phi_{12}=m \pi,
$$

so that $\epsilon=\epsilon^{\prime}(\Delta \phi)+\epsilon(\Delta l)$, where

$\epsilon^{\prime}(\Delta \phi)=\Delta \phi\left\{2 l_{1} / \lambda\left[1-\left(\phi_{c 1} / \phi_{0}^{\prime}\right)^{2}\right]^{1 / 2}+d \phi_{12}\left(\phi_{0}^{\prime}\right) / d \phi\right\}$,

and where $\epsilon(\Delta l)$ is still given by Eq. $\left(23^{\prime}\right)$. The variation of $\epsilon^{\prime}(\Delta \phi)$ with $\Delta \phi$ is now much sharper because $\phi_{0}^{\prime} \sim \phi_{c 1}$, leading to a large first contribution (the $\hat{\mathbf{z}}$ component of the refracted wave vector $g_{1}^{\prime}$ rapidly increases for $\left.\phi \geq \phi_{c 1}\right)$, and because the additional phase contribution $\phi_{12}(\phi)$ rapidly increases from $-\pi \rightarrow 0$ as $\phi \propto \phi_{c 1} \rightarrow \phi_{c 2}$. However, since the damping-stablized film is thicker than the corresponding $\lambda / 4$ film, $\epsilon(\Delta l)$ is smaller, so dampingstabilized films are less sensitive to film thickness variations.

The effects of beam divergence and long-range thickness variations are shown in Fig. 10 for the various GIAR films of $\mathrm{Te}$ on $\mathrm{Fe}$. Here the solid curves give the rocking curves $|R|^{2}$ vs $\phi$ for the idealized cases of $\Delta \phi=\Delta l=0$, while the dashed curves near the principal minimum are calculated for a beam spread of $\Delta \phi=0.2 \mathrm{mrad}$ and a $5 \%$ thickness variation. We note that the damping-stabilized minima and the $3 \lambda / 4$ minimum are strongly affected, now being only $|R|^{2} \approx 10^{-2}-10^{-1}$, while the $\lambda / 4$ minimum is still quite pronounced, $|R|^{2} \approx 8 \times 10^{-4}$.

In Table III we give average reflectivities for several 
TABLE III. Optimal parameters $l_{1}, \phi_{0}$ and minimum reflectivities for several coatings on $\mathrm{Fe}$ reflecting 14.4-keV radiation. The response is averaged over an incident beam spread of 0.25 $\mathrm{mrad}$ and over a long-range film thickness variation of $l_{1} \pm 0.025 l_{1}$.

\begin{tabular}{lcccc}
\hline \hline & $\begin{array}{c}\phi_{0} \\
(\mathrm{mrad})\end{array}$ & $\begin{array}{c}l_{1} \\
(\AA)\end{array}$ & $|R|^{2 \mathrm{a}}$ & $|R|_{\mathrm{Fe}}^{2} /|R|^{2}$ \\
\hline $\mathrm{Ti}$ & 6.02 & 42 & $2.4(-5)$ & 719 \\
$\mathrm{Se}$ & 6.03 & 40 & $2.1(-5)$ & 807 \\
$\mathrm{Ge}$ & 4.57 & 65 & $3.7(-4)$ & 245 \\
$\mathrm{Te}$ & 4.38 & 76 & $9.4(-4)$ & 135 \\
$\mathrm{Ga}$ & 4.21 & 85 & $2.7(-3)$ & 68 \\
$\mathrm{~V}$ & 4.19 & 93 & $3.5(-3)$ & 54 \\
$\mathrm{Sb}$ & 4.15 & 97 & $5.2(-3)$ & 41 \\
$\mathrm{Ce}$ & 4.12 & 98 & $7.2(-3)$ & 32 \\
$\mathrm{Zr}$ & 4.17 & 96 & $4.7(-3)$ & 40 \\
\hline
\end{tabular}

The notation $(-x)$ denotes $\times 10^{-x}$.

$\lambda$ /4-GIAR films on $\mathrm{Fe}$, averaged over a beam divergence of $0.25 \mathrm{mrad}$ and over a variation in film thickness $l_{1} \pm 0.025 l_{1}$. (The optimal parameters $l_{1}, \phi_{0}$ are shifted somewhat by finite $\Delta \phi, \Delta l$ averages.) We note in particular that strong suppressions are still possible, with, e.g., reduction factors $|R|^{2} /|R|_{\text {Fe }}^{2}$ of $\frac{1}{807}$ for Se, $\frac{1}{245}$ for $\mathrm{Ge}$, and $\frac{1}{135}$ for $\mathrm{Te}$, corresponding to minimum reflectivities in the range $|R|^{2} \simeq 10^{-5}-10^{-3}$.

\section{Frequency range of suppression}

For incident "white" synchrotron radiation, an important question is the frequency region over which suppression occurs. For $\lambda / 4-$ GIAR films, one effect of a frequency variation is again the change of the quarter-wave condition, due both to the direct change in $\lambda$ and to the altered refraction angle (produced by the change in critical angle $\phi_{c 1}$, which is linear in $\lambda$ ). For a film which is a $\lambda / 4$-GIAR film for the parameters $\lambda_{0}, l_{1}, \phi_{0}$, a wavelength variation $\lambda_{0} \rightarrow \lambda_{0}+\Delta \lambda$ changes the phase to

$$
2 l_{1} \phi_{0}\left[1-\left(\phi_{c 1} / \phi_{0}\right)^{2}\right]^{1 / 2} / \lambda=m \pi+\epsilon(\Delta \lambda),
$$

where

$$
\epsilon(\Delta \lambda)=-\frac{\Delta \lambda}{\lambda_{0}}\left(m \pi\left\{1+\phi_{c 1}\left(\lambda_{0}\right)^{2} /\left[\phi_{0}^{2}-\phi_{c 1}\left(\lambda_{0}\right)^{2}\right]\right\}\right) .
$$

As before, for $|\epsilon(\Delta \lambda)| \lesssim 0.2$, there is very strong suppression, typically $|R|^{2} \leq 10^{-3}$. For the 80 - $\AA$ GIAR film of $\mathrm{Te}$ on $\mathrm{Fe}, \mid \epsilon(\Delta \lambda) \tilde{\mid} \lesssim 0.2$ for $|\Delta \lambda| / \lambda_{0} \lesssim 0.04$,

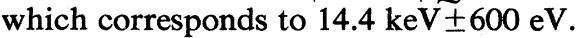

A frequency variation also effects the impedance-match condition because the critical angles $\phi_{c 1}, \phi_{c 2}$ are changed as $\lambda_{0} \rightarrow \lambda_{0}+\Delta \lambda$, which changes the reflectivities $r_{01}$ and $r_{12}$. Because $\phi_{c}$ is linear in $\lambda$ [Eq. (5)], the resulting mismatch is very nearly that produced by an effective angular variation $\Delta \phi=\left(\Delta \lambda / \lambda_{0}\right) \phi_{c}$. For the $80-\AA$ Te GIAR film, $\left|r_{01}-r_{12} \exp \left(-2 g_{1}^{\prime \prime} l_{1}\right)\right|^{2} \lesssim 10^{-3}$ over an angular region $\Delta \phi \simeq 0.4 \mathrm{mrad}$, which corresponds to a wavelength variation $\Delta \lambda / \lambda_{0} \simeq \Delta \phi / \phi_{c} \simeq 0.1$, again about $1 \mathrm{keV}$ wide.

The conclusion then is that a $\lambda / 4$-GIAR film (lowest order) will give a strong suppression to reflection over about a $10 \%$ bandwidth, corresponding to $\approx 1 \mathrm{keV}$ in the

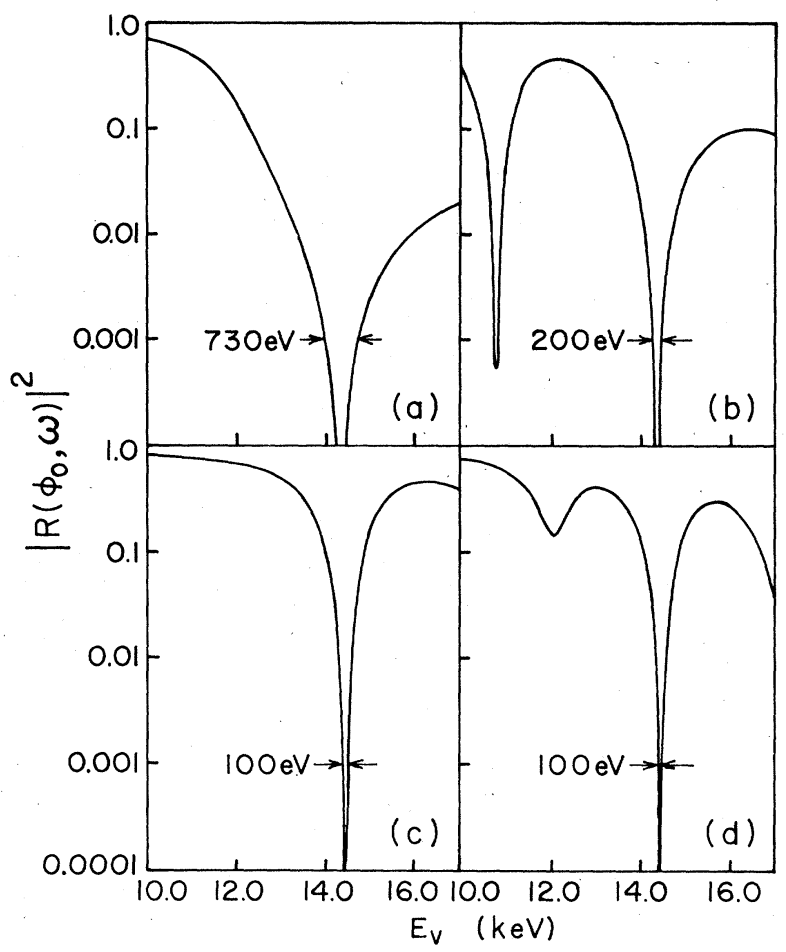

FIG. 12. $|R|^{2}$ vs $\omega$ for the four possible GIAR films of Te on $\mathrm{Fe}$.

$10-\mathrm{keV}$ region. For higher-order films the band will be narrower. Figures $12(\mathrm{a})$ and $12(\mathrm{~b})$ give $|R|^{2}$ vs $\omega$ for the 80- and $268-\AA \lambda / 4-G I A R$ films of $\mathrm{Te}$ on Fe. Here, $|R|^{2}<10^{-3}$ over the regions $\Delta \omega \approx 730$ and $200 \mathrm{eV}$, respectively.

For the damping-stabilized film, on the other hand, the phase and impedance-match conditions will vary much more rapidly as $\lambda_{0} \rightarrow \lambda_{0}+\Delta \lambda$, just as for angular variations and consequently the frequency region of strong suppression will be narrowed. This is shown explicitly in Figs. 12(c) and 12(d) for the 240- and 409- $\AA$ dampingstabilized GIAR films of $\mathrm{Te}$ on $\mathrm{Fe}$. However, for $|R|^{2} \lesssim 0.05$, which is a typical reflectivity from a damping-stabilized GIAR film when averaged over thickness variations and beam divergence, $\Delta \omega \approx 600 \mathrm{eV}$.

\section{SUMMARY}

The purpose of this paper has been to develop the basic theory and to examine the simplest techniques for producing grazing-incidence antireflection films to suppress the reflection of $1-\AA \times$ rays and to examine the general response of the films - the degree of suppression, the frequency range of suppression, and the limitations imposed by beam divergence and long-range variations in film thickness. Our main conclusions are as follows.

For a mirror coated with a single film, there are two possible antireflection coatings-a $\lambda / 4-G I A R$ film $\left(\phi_{0}>\phi_{c 2}\right)$ or a damping-stabilized GIAR film $\left(\phi_{c 1}<\phi_{0}<\phi_{c 2}\right)$. In either case the electron density $\rho_{1}$ of the film must be less than that of the mirror and, for a given $\rho_{1}<\rho_{2}$, the required film thickness $l_{1}$ and the 
operating angle $\phi_{0}$ for a GIAR film are then uniquely defined.

For a $\lambda / 4$-GIAR film, significant changes in the reflectivity occur for angular variations of $\Delta \phi \sim 0.1 \phi_{c} \sim 10^{-4}$ $\mathrm{rad}$ and for film thickness variations of $\Delta l \sim 0.1 \lambda / \phi \sim 10$ A. When the response is averaged over long-range variations in film thickness of $\Delta l \approx 10 \AA$ and angular variations of $\Delta \phi \approx 10^{-4}$ rad, reduced reflectivities of order $10^{-4} \leqslant|R|^{2} \leqslant 10^{-3}$ should be possible over about a $\Delta \omega \approx \mathbb{1}-\mathrm{keV}$ frequency range. For damping-stabilized GIAR films, the angular response is sharper but there is a greater $\Delta l$ tolerance, giving averaged reflectivities of order $10^{-2} \lesssim|R|^{2} \lesssim 10^{-1}$ over a $\Delta \omega \approx 0.5-\mathrm{keV}$ range.

\section{ACKNOWLEDGMENTS}

This work was partially supported by the National Science Foundation Grant No. DMR-80-15706, and the Deutsche Forschungsgemeinschaft and the Bundesministerium für Forschung und Technologie Grant No. 05269GU.

\section{APPENDIX A}

Here we briefly outline the dynamical solution for grazing-incidence reflection of $\gamma$ rays from a mirror coated with one or more films, and we also comment on the essential equivalence of this approach to the index-ofrefraction solution.

$\gamma$-ray (or $\mathrm{x}$-ray) optics is a single-photon optics governed by simple multiple-scattering equations of a semiclassical form [Eqs. (50) and (51) of Ref. 27]. From these equations, two equivalent formulations of the dynamical theory are possible-a generalized DarwinPrins planar solution, or a Laue solution. ${ }^{32}$ Here we follow the Darwin-Prins solution.

As discussed in Refs. 26 and 27, grazing incidence can be viewed as a special case of Bragg reflection $\left(\phi_{B}=0\right)$. Conceptually dividing film 1 into $M$ layers of thickness $d=l_{1} / M \rightarrow 0$, the dynamical equations are given as

$$
\begin{aligned}
& \mathbf{T}_{m}=e^{i g_{0} d}\left[\left(1+i \widetilde{F}^{t t}\right) \mathbf{T}_{m-1}+i \widetilde{F}^{t r} \mathbf{R}_{m-1}\right], \\
& \mathbf{R}_{m}=e^{i g_{0} d}\left[\left(1+i F^{r r}\right) \mathbf{R}_{m+1}+i \widetilde{F}^{r t} T_{m+1}\right] .
\end{aligned}
$$

Here the notation is that of Ref. 27: $\mathbf{T}_{m}$ is the wave $[(\mathbf{k}, \omega)$ Fourier component] incident from above on the $m$ th layer in the incident $\mathbf{k}_{t}=\mathbf{k}_{0}$ channel [see Fig. 13(a)], $\mathbf{R}_{m}$ is the wave incident from below on the $m$ th layer in the reflected $\mathbf{k}_{r}$ channel, and $i \widetilde{F^{s s^{\prime}}}$ is the dimensionless $2 \times 2$ planar reflection amplitude for scattering $\mathbf{k}_{s^{\prime}}$ radiation into $\mathbf{k}_{s}$ radiation. The matrix elements of $\widetilde{F}^{s s^{\prime}}$ are explicitly $F_{a b}^{s s^{\prime}}=n \lambda d f\left(\mathbf{k}_{s}, \widehat{\boldsymbol{\epsilon}}_{a}^{s} ; \mathbf{k}_{s^{\prime}}, \widehat{\boldsymbol{\epsilon}}_{b}^{s^{\prime}}\right) / \phi_{0}$, where $f$ is the atomic scattering amplitude [given by Eq. (4) for forward scattering].

As indicated schematically in Fig. 13(b), the first relation in (A1) simply states that the total wave $\left(\mathbf{T}_{m}\right)$ incident on the $m$ th layer from above in the $t$ channel is equal to the total amplitude $\left(\mathbf{T}_{m-1}\right)$ incident on the $m-1$ layer plus the forward scattering of this wave by the $m-1$ layer $\left(i \widetilde{F}^{t t} T_{m-1}^{(t)}\right.$ ) plus the amplitude $\left(i F^{t r} R_{m-1}\right)$ of the wave scattering from the $r$ channel to the $t$ channel by

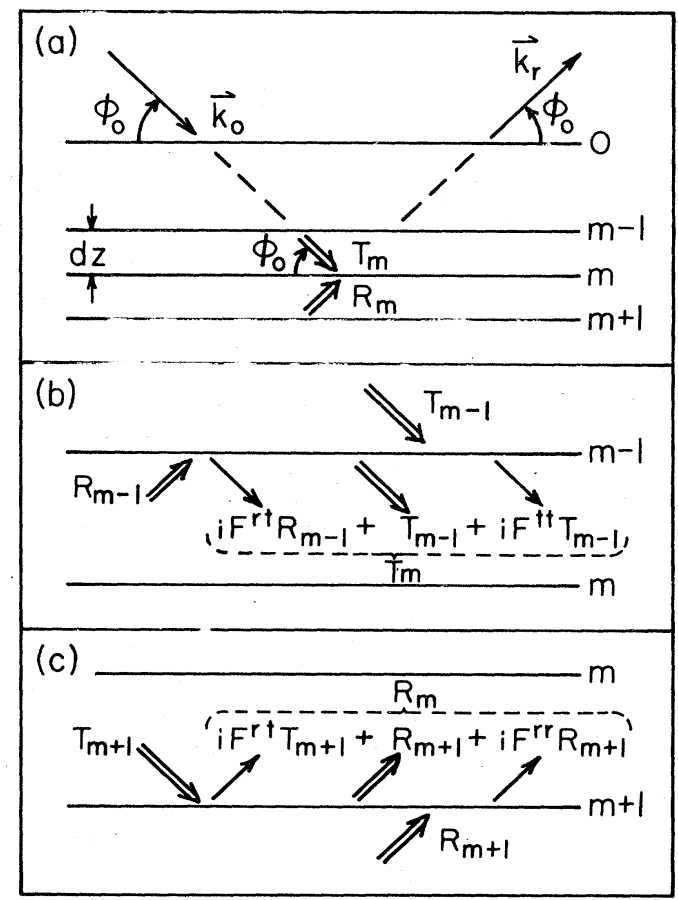

FIG. 13. (a) Schematic representation of "planar" breakup of film 1. Here the $m$ th "plane" represents a differential layer of thickness $d z=l_{1} / M \rightarrow 0$. (b) Relation between $T_{m}$ and $T_{m-1}, R_{m-1}$. (c) Relation between $R_{m}$ and $R_{m+1}, T_{m+1}$.

the $(m-1)$ layer. All these effects are propagated to the $m$ th layer by the phase factor $e^{i g_{0} d}$. Similarly, the second relation in (A1) relating $R_{m}$ to the $r$-and $t$-channel waves at the $m+1$ layer has the simple interpretation indicated in Fig. 13(c).

For grazing incidence, $\widetilde{F}^{t r}=\widetilde{F}^{r t}=\widetilde{F}^{r r}=\widetilde{F}^{t t} \equiv F$ to terms of order $\phi .{ }^{27}$ With this approximation, the optical equations can be simultaneously diagonalized. If film 1 is nonresonant, then any polarization basis will diagonalize the equations. More generally we will assume that either film 1 or one of the lower layers is resonant. Then for incident $\widehat{\boldsymbol{\epsilon}}_{\eta}$ eigenpolarization of the resonant medium, ${ }^{33}$ the basic equations for the wave amplitudes in film 1 are now the scalar equations

$$
\begin{aligned}
& T_{m}^{\eta}=e^{i g_{0} d}\left\{[1+i F(\eta)] T_{m-1}^{\eta}+i F(\eta) R_{m-1}^{\eta}\right\} \\
& R_{m}^{\eta}=e^{i g_{0} d}\left\{[1+i F(\eta)] R_{m+1}^{\eta}+i F(\eta) T_{m+1}^{\eta}\right\}
\end{aligned}
$$

These finite-difference equations can be converted to differential equations with $d \rightarrow d z, T_{m} \rightarrow T(z), R_{m}$ $\rightarrow R(z)$, and $F \rightarrow G d z\left[G=n \lambda f(\eta) / \phi_{0}\right]$, giving

$$
\begin{aligned}
& \frac{d T(z)}{d z}=i\left(g_{0}+G\right) T(z)+i G R(z), \\
& -\frac{d R(z)}{d z}=i\left(g_{0}+G\right) R(z)+i G T(z),
\end{aligned}
$$

where here we have suppressed the polarization index $\eta$. These equations have the immediate solutions, 


$$
\begin{aligned}
& T(z)=t_{(+)} e^{i g_{1} z}+t_{(-)} e^{-i g_{1} z}, \\
& R(z)=r_{(+)} e^{i g_{1} z}+r_{(-)} e^{-i g_{1} z},
\end{aligned}
$$

where $g_{1}=\left[g_{0}\left(g_{0}+2 G\right)\right]^{1 / 2}=k \phi_{0} \beta_{1}$ as given by Eq. (3).

For a unit incident amplitude $\hat{\boldsymbol{\epsilon}}_{\eta}$, the boundary condition at the upper surface is simply

$$
T(z=0)=1 \text {, }
$$

while at the interface of film 1 and medium 2 the boundary condition is

$$
R\left(z=l_{1}\right)=R_{02}^{\dagger}(\eta) T\left(z=l_{1}\right) \text {. }
$$

Here, $R_{02}^{\dagger}(\eta)$ is the total vacuum-medium reflection amplitude of medium 2 for incident $\hat{\boldsymbol{\epsilon}}_{\eta}$ eigenpolarization. If medium 2 is infinite, then the dynamical theory gives the usual expression

$$
R_{02}^{\dagger}(\eta)=R_{02}(\eta)=\frac{1-\beta_{2}(\eta)}{1+\beta_{2}(\eta)} .
$$

The two boundary conditions (A5) and (A6) plus the two optical equations (A3) uniquely determine the coefficients $r_{( \pm)}, t_{( \pm)}$in (A4). After some manipulation, ${ }^{34}$ the reflection amplitude is then given by

$R=R(z=0)=r_{(+)}+r_{(-)}=\frac{R_{01}+R_{12} e^{i 2 g_{1} l_{1}}}{1+R_{01} R_{12} e^{i 2 g_{1} l_{1}}}$,

in agreement with Eq. (9).

For several films the solution can be obtained by iteration: For example, for a medium 3 coated with two films, 1 and 2, the boundary condition at the 1-2 interface is again given by (A6) but now with $R_{02}^{\dagger}$ given by (A8) (with the indices changes $1 \rightarrow 2,2 \rightarrow 3$ ). The resulting solution for two films is, of course, just that given by Eq. (12).

Although these results agree with the index-ofrefraction results, the dynamical theory still has a somewhat different appearance. In particular, for a single infinite media $\left(l_{1} \rightarrow \infty\right)$, the dynamical theory is still a twowave theory with internal $t$ - and $r$-channel waves,

$$
\begin{aligned}
& T(z)=t_{(+)} e^{i g_{1} z}, \\
& R(z)=r_{(+)} e^{i g_{1} z},
\end{aligned}
$$

with $t_{(+)}=1, r_{(+)}=\left(1-\beta_{1}\right) /\left(1+\beta_{1}\right)\left(r_{(-)}=t_{(-)}=0\right.$ in the limit $\left.l_{1} \rightarrow \infty\right)$, whereas in the index-of-refraction ap-

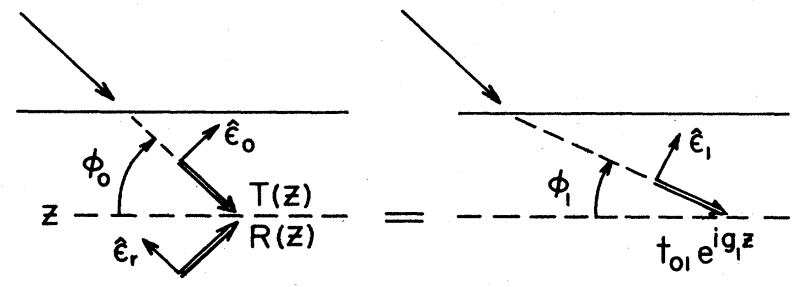

FIG. 14. Equivalence of two-wave dynamical treatment and the single-wave refraction treatment. proach there is a single internal wave,

$$
\mathbf{A}_{T}(z)=\widehat{\boldsymbol{\epsilon}}_{1} t_{01} e^{i g_{1} z},
$$

with $t_{01}=2 /\left(1+\beta_{1}\right)$ (see Fig. 14). There is of course no discrepancy here: In the dynamical theory the total wave field at $z$ is in fact a wave traveling in the refracted direction $\mathbf{k}_{1}$, with polarization $\hat{\boldsymbol{\epsilon}}_{1}$ and with the refraction angle $\phi_{1}$ determined by Snell's law $N_{1} \cos \phi_{1}=\cos \phi_{0}$, i.e., by direct substitution from (A9),

$$
\begin{aligned}
\mathbf{A}_{T}(z) & \equiv \hat{\boldsymbol{\epsilon}}_{0} T(z)+\hat{\boldsymbol{\epsilon}}_{r} R(z) \\
& =\hat{\boldsymbol{\epsilon}}_{1} t_{01} e^{i g_{1} z}\left[1+O\left(1-N_{1}\right)\right] .
\end{aligned}
$$

Here the correction terms are of order $\left(1-N_{1}\right) \approx 10^{-5}$, which is the same order of magnitude of the other approximations in the theory.

\section{APPENDIX B}

There is extensive literature on thin-film interference techniques for optical reflections from thin dielectric films ${ }^{1-6}$ and these ideas can be taken over directly to $x-$ ray grazing incidence (within the limits imposed by strong photoabsorption). However, almost all the theory for thin-film optical filters is expressed in terms of the "characteristic matrices" $\widetilde{\mathscr{M}}_{n}$ which relate the tangential $E$ and $B$ fields at the adjacent boundaries of the $n$th film.

In order to make this literature more accessible to our case, we summarize the main results, making the appropriate modifications for $\mathrm{x}$-ray grazing incidence. For nonmagnetic dielectric films, the tangential components of $\mathbf{E}$ and $\mathbf{B}$ are continuous across each boundary. For the $m$ th film, $E_{m}$ and $B_{m}$ designate the tangential components of the total $\mathbf{E}$ and $\mathbf{B}$ fields at the upper interface (see Fig. 15) and $E_{m+1}, B_{m+1}$ are the fields at the lower interface. It is straightforward to show that the fields at adjacent boundaries are related by ${ }^{1,4}$

$$
\left[\begin{array}{l}
E_{m} \\
B_{m}
\end{array}\right]=\widetilde{\mathscr{M}}_{m}\left(\begin{array}{l}
E_{m+1} \\
B_{m+1}
\end{array}\right),
$$

where $\widetilde{\mathscr{M}}_{m}$ is the characteristic matrix for the $m$ th film,

$$
\tilde{\mathscr{M}}_{m}=\left(\begin{array}{cc}
\cos \delta_{m} & i \sin \delta_{m} / \eta_{m} \\
i \eta_{m} \sin \delta_{m} & \cos \delta_{m}
\end{array}\right]
$$

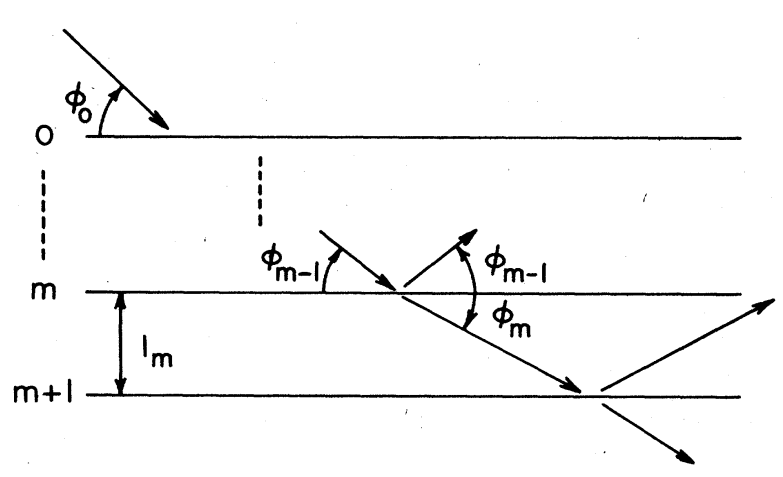

FIG. 15. Multilayer film geometry. 
and where here

$$
\delta_{m}=k_{0} l_{m} N_{m} \sin \phi_{m} \simeq g_{m} l_{m},
$$

with $g_{m}=k_{0} \phi_{0} \beta_{m}$ as given in Eqs. (2) and (3), and

$$
\begin{aligned}
& \eta_{m}^{(\sigma)}=N_{m} \sin \phi_{m} \simeq \phi_{0} \beta_{m}, \\
& \eta_{m}^{(\pi)}=N_{m} / \sin \phi_{m} \simeq\left(\phi_{0} \beta_{m}\right)^{-1},
\end{aligned}
$$

for $\sigma$ and $\pi$ polarization, respectively. In (B3) and (B4), $N_{m}=1+2 \pi \hbar^{2} n f \simeq\left(1-10^{-5}\right)$ is the index of refraction of the $m$ th film, and to get the final form, we have used Snell's law for parallel interfaces, $N_{m} \cos \phi_{m}=N_{0} \cos \phi_{0}$.

For a series of $p$ films plus substrate $s$, the characteristic matrix $\widetilde{\mathscr{M}}$ for the entire series (which relates the total $E_{0}$ and $B_{0}$ at the top surface to $E_{p+1}=E_{s}, B_{p+1}=B_{s}$ at the substrate surface) is then

$$
\tilde{\mathscr{M}}=\tilde{\mathscr{M}}_{1} \cdots \tilde{\mathscr{M}}_{p}=\left(\begin{array}{ll}
m_{11} & m_{12} \\
m_{21} & m_{22}
\end{array}\right]
$$

and the amplitude of the reflected wave is given by ${ }^{4}$

$$
R=\frac{\eta_{0} m_{11}+\eta_{0} \eta_{s} m_{12}-m_{21}-\eta_{s} m_{22}}{\eta_{0} m_{11}+\eta_{0} \eta_{s} m_{12}+m_{21}+\eta_{s} m_{22}}
$$

where $\eta_{0}\left(=\phi_{0}\right.$ or $\left.\phi_{0}^{-1}\right)$ and $\eta_{s}$ are defined by (B4). Thus, to get $R$ for any configuration of films, the procedure is simply to calculate $\widetilde{\mathscr{M}}_{m}$ for each film, multiply in sequential order to get $\widetilde{\mathscr{M}}$, and substitute into (B6).

This is a straightforward procedure and gives a closed expression for any series of films. Furthermore, in the absence of absorption, the character matrix $\widetilde{\mathscr{M}}_{m}$ is very sim- ple for a half-wave film $\left[\delta_{m}=(2 n+1) \pi\right.$ and $\widetilde{\mathscr{M}}_{m}$ is diagonal and equal to -1$]$ or for a quarter-wave film $\left[\delta_{m}=(2 n+1) \pi / 2\right.$ and the diagonal elements of $\widetilde{\mathscr{M}}_{m}$ are zero], which makes the analysis for a series of half- and quarter-wave films particularly easy. The disadvantage of this approach is that the physics is obscured and, moreover, in the presence of strong absorption, the special simplicity of $\widetilde{\mathscr{M}}$ for half- and quarter-wave films is lost.

As a particular example of the formalism for a single film $\underline{1}$ on a substrate $\underline{2}, \widetilde{\mathscr{M}}=\widetilde{\mathscr{M}}_{1}$ is given by (B2), $\eta_{0}=\phi_{0}$, $\eta_{s}=\phi_{0} \beta_{2}$ (taking $\sigma$ polarization), giving

$$
R=\frac{\cos \left(g_{1} l_{1}\right)\left(1-\beta_{2}\right)+i \sin \left(g_{1} l_{1}\right)\left(\beta_{2} / \beta_{1}-\beta_{1}\right)}{\cos \left(g_{1} l_{1}\right)\left(1+\beta_{2}\right)+i \sin \left(g_{1} l_{1}\right)\left(\beta_{2} / \beta_{1}+\beta_{1}\right)} .
$$

With some manipulation, Eq. (B7) can be shown to be equivalent to Eq. (9). The condition for an antireflection film $R=0$ gives the condition

$$
\tan \left(g_{1} l_{1}\right)=-i\left(1-\beta_{2}\right) /\left(\beta_{2} / \beta_{1}-\beta_{1}\right),
$$

which has no obvious interpretation. However, by making use of $\tanh ^{-1} z=\ln \sqrt{(1+z) /(1-z)}$, the condition can be transformed to

$$
\operatorname{ig}_{1} l_{1}=\frac{1}{2} \ln \left(-R_{01} / R_{12}\right) \text {, }
$$

where $R_{01}, R_{12}$ are the interface reflection amplitudes. The real and imaginary parts of (B9) give the impedancematch and destructive interference conditions of Eqs. (20) and (22). Either approach leads to the same results, but the interface reflection approach is more intuitive.
${ }^{1}$ H. A. Macleod, Thin Film Optical Filters (American Elsevier, New York, 1969) and references therein.

2J. T. Cox and G. Hass, in Physics of Thin Films, edited by G. Hass and R. E. Thun (Academic, New York, 1964), Vol. 2, pp. 239-304.

${ }^{3}$ A. Vasicek, Optics of Thin Films (North-Holland, Amsterdam, 1960).

${ }^{4}$ E. Hecht and A. Zajac, Optics (Addison-Wesley, Reading, Mass., 1974), pp. 311-316.

${ }^{5} \mathrm{M}$. Born and E. Wolf, Principles of Optics (Pergamon, New York, 1959), pp. 50-69.

${ }^{6}$ P. Bowmeister and G. Pincus, Sci. Am. 223, 59 (1970).

${ }^{7}$ E. Spiller, Appl. Phys. Lett. 20, 365 (1972).

${ }^{8}$ R.-P. Haelbich and C. Kunz, Opt. Commun. 17, 287 (1976).

${ }^{9}$ A. V. Vinogradov and B. Ya. Zeldovich, Appl. Opt. 16, 89 (1977).

${ }^{10}$ R.-P. Haelbich, A. Segmüller, and E. Spiller, Appl. Phys. Lett. 34, 184 (1979).

${ }^{11}$ E. Spiller, A. Segmüller, J. Rife, and R.-P. Haelbich, Appl. Phys. Lett. 37, 1048 (1980).

${ }^{12}$ F. Mezei, Commun. Phys. 1, 81 (1976); 2, 41(E) (1977).

${ }^{13}$ A. M. Saxena and B. P. Schoenborn, Acta Crystallogr. Sect. A 33, 805 (1977).

14J. P. Hannon, G. T. Trammell, M. Mueller, E. Gerdau, H. Winkler, and R. Rüffer, Phys. Rev. Lett. 43, 636 (1979).

${ }^{15}$ A. H. Compton, Philos. Mag. 45, 1121 (1923).

${ }^{16}$ H. Kiessig, Ann. Phys. (Leipzig) 10, 715 (1931); 10, 769 (1931).

${ }^{17}$ A. I. Alichanow and L. A. Arzimowic, Z. Phys. 82, 489
(1933).

${ }^{18}$ L. G. Parratt, Phys. Rev. 95, 359 (1954).

${ }^{19}$ L. M. Rieser, J. Opt. Soc. Am. 47, 987 (1957).

${ }^{20}$ A. Segmüller, Thin Solid Films 18, 287 (1973).

21 J. P. Hannon, N. V. Hung, G. T. Trammell, E. Gerdau, M. Mueller, R. Rüffer, and H. Winkler, following paper (II), Phys. Rev. B 32, 5081 (1985).

22J. P. Hannon et al., paper III, Phys. Rev. B (to be published).

23J. P. Hannon et al., paper IV, Phys. Rev. B (to be published).

${ }^{24}$ J. P. Hannon, J. T. Hutton, G. T. Trammell, E. Gerdau, and R. Rüffer, paper V (unpublished).

25J. P. Hannon, J. T. Hutton, G. T. Trammell, E. Gerdau, and R. Rüffer, paper VI (unpublished).

${ }^{26} \mathrm{G}$. T. Trammell, Chemical Effects of Nuclear Transformations (International Atomic Energy Agency, Vienna, 1961), Vol. 1, p. 75.

27J. P. Hannon and G. T. Trammell, Phys. Rev. 186, 306 (1969).

28J. P. Hannon, N. J. Carron, and G. T. Trammell, Phys. Rev. B 9, 2810 (1974), p. 2828

${ }^{29}$ E.g., R. W. James, The Optical Principles of the Diffraction of $X$-rays (Cornell University Press, Ithaca, 1965), pp. 167, 171190, and 426-435.

${ }^{30} \mathrm{G}$. Materlik (private communication).

${ }^{31} \mathrm{M}$. Harsdorff (private communication).

${ }^{32}$ See Appendix D of Ref. 27.

${ }^{33}$ For the explicit expression for the eigenpolarizations, see Refs. 22 or 27.

${ }^{34}$ Here it is useful to note the addition rule that $\left(R_{10}+R_{02}\right) /\left(1+R_{10} R_{02}\right)=R_{12}$ 